This is a self-archived version of an original article. This version may differ from the original in pagination and typographic details.

Author(s): Kauranen, Aapo; Koskela, Pekka; Zapadinskaya, Aleksandra

Title: Regularity and modulus of continuity of space-filling curves

Year: 2019

Version: Accepted version (Final draft)

Copyright: (C) The Hebrew University of Jerusalem 2019

Rights: In Copyright

Rights url: http://rightsstatements.org/page//nC/1.0/?language=en

Please cite the original version:

Kauranen, A., Koskela, P., \& Zapadinskaya, A. (2019). Regularity and modulus of continuity of space-filling curves. Journal d'Analyse Mathematique, 137(1), 73-100.

https://doi.org/10.1007/s11854-018-0066-3 


\title{
REGULARITY AND MODULUS OF CONTINUITY OF SPACE-FILLING CURVES
}

\author{
AAPO KAURANEN AND PEKKA KOSKELA AND ALEKSANDRA ZAPADINSKAYA
}

\begin{abstract}
АвstRACт. We study critical regularity assumptions on space-filling curves that possess certain modulus of continuity. The bounds we obtain are essentially sharp, as demonstrated by an example.
\end{abstract}

\section{INTRODUCTION}

In 1890, G. Peano [15] gave the first example of a curve whose range contains the entire unit square. More precisely, he constructed a continuous mapping $g:[0,1] \rightarrow \mathbb{R}^{2}$, such that $[0,1]^{2} \subset g([0,1])$. Mappings like this are now customarily called Peano curves. Peano's construction allowed also for space-filling: there exists a continuous mapping $g:[0,1] \rightarrow \mathbb{R}^{n}$, where $n \in \mathbb{N}, n \geq 3$, such that $[0,1]^{n} \subset g([0,1])$. In what follows, we call such a $g$ a spacefilling curve. Once these mappings are known to exist, it is natural to ask how regular they can be. Regularity properties of space-filling curves can be measured in terms of continuity and total oscillation (energy). Despite the age of the topic the research is still active. For some recent advances see [6], [17], [3], [18], [19]. In this paper we consider the interplay of modulus of continuity and energy conditions and our results are not covered by previous results.

For technical reasons, let us consider the case of closed curves $g: S^{1} \rightarrow \mathbb{R}^{n}, n \geq 2$, parameterized by the unit circle $S^{1}$. Regarding continuity, a simple dimension estimate shows that a Peano curve cannot be Hölder continuous with any exponent strictly greater that $1 / 2$, and similarly with $1 / n$ in the case of a space-filling curve. Hölder continuity exponents $1 / 2$ and $1 / n$ are actually possible, see, for instance $[1,6,17]$. Moreover, it is possible to construct an almost everywhere differentiable Peano (space-filling) curve with Hölder continuity exponent arbitrarily close to $1 / 2(1 / n)$ (see [16, Chapter 5] for one of such constructions).

One is then motivated to ask whether a suitable energy bound would rule out space filling. Indeed, if the component functions of $g: S^{1} \rightarrow \mathbb{R}^{n}$ are absolutely continuous, then $g\left(S^{1}\right)$ necessarily has finite length; hence it cannot cover a square or a cube. Thus, the case of usual Sobolev regularity is trivial, and one is lead to consider fractional derivatives. The component functions of $g: S^{1} \rightarrow \mathbb{R}^{2}$ belong to the fractional Sobolev space $W^{\frac{1}{2}, 2}\left(S^{1}\right)$ exactly when the Poisson extension $P g: B(0,1) \rightarrow \mathbb{R}^{2}$ belongs to the Sobolev space $W^{1,2}\left(B(0,1) ; \mathbb{R}^{2}\right)$. Such Peano curves do exist [12, Section 5], but according to [12, Theorem D], $P g$ cannot be Hölder continuous (hence neither can $g$ ) under this regularity of $P g$. Thus, Hölder continuity

2010 Mathematics Subject Classification. 26A15, 26A33, 28 A78.

The research of Aapo Kauranen and Pekka Koskela have been supported by the Academy of Finland via the Centre of Excellence in Analysis and Dynamics Research (project No. 271983). The research of Aleksandra Zapadinskaya has been partially supported by the Academy of Finland, by the University of Cincinnati and by "Scuola Galileo Galilei" research grant at the University of Pisa. 
and finiteness of a suitable energy prevent $g$ from covering a square, actually force $g\left(S^{1}\right)$ to be of area zero. The energy in question is the one corresponding to the Sobolev space $W^{\frac{1}{2}, 2}\left(S^{1}\right)$.

From above, we see that neither Hölder continuity nor finiteness of $W^{\frac{1}{2}, 2}\left(S^{1}\right)$-energy is sufficient to rule out square filling, but together they are. Let us analyze the energy assumption in more detail. Given $g: S^{1} \rightarrow \mathbb{R}^{2}$, this energy is essentially

$$
\int_{S^{1}} \int_{S^{1}} \frac{|f(x)-f(y)|^{2}}{|x-y|^{2}} d \mathcal{H}_{x}^{1} d \mathcal{H}_{y}^{1} .
$$

For our purposes, we consider a dyadic version of this energy, given by

$$
\mathcal{E}(g ; 2,0):=\sum_{i=1}^{\infty} \sum_{j=1}^{2^{i}}\left|g_{i_{i, j}}-g_{U_{i, j}}\right|^{2} .
$$

Here, $\left\{I_{i, j}: i \in \mathbb{N}, j=1, \ldots, 2^{i}\right\}$ is a dyadic decomposition of $S^{1}$, such that for a fixed $i \in \mathbb{N}$, $\left\{I_{i, j}: j=1, \ldots, 2^{i}\right\}$ is a family of arcs of length $2 \pi / 2^{i}$ with $\cup_{j} I_{i, j}=S^{1}$. The next generation is constructed in such a way that for each $j \in\left\{1, \ldots, 2^{i+1}\right\}$, there exists a unique number $k \in\left\{1, \ldots, 2^{i}\right\}$, satisfying $I_{i+1, j} \subset I_{i, k}$. We denote this parent of $I_{i+1, j}$ by $U_{i+1, j}$, and $U_{1, j}=S^{1}$ for $j=1,2$. By $g_{A}, A \subset S^{1}$, we denote the mean value $g_{A}=f_{A} g d \mathcal{H}^{1}=\frac{1}{\mathcal{H}^{1}(A)} \int_{A} g d \mathcal{H}^{1}$. One can check that finiteness of the former energy implies the finiteness of the latter (see Theorem 3.1).

If $g: S^{1} \rightarrow \mathbb{R}^{2}$ is a 1/2-Hölder continuous Peano curve, then the inner sum in (1) is uniformly bounded from above. Additionally, one can rather easily obtain a positive lower bound for the inner sum in the case of a certain almost everywhere differentiable Peano curve with Hölder exponent arbitrarily close to $1 / 2$. Thus the energy (1) does not appear critical for Hölder continuous maps. Let us consider

$$
\mathcal{E}(g ; 2, \lambda):=\sum_{i=1}^{\infty} i^{\lambda} \sum_{j=1}^{2^{i}}\left|g_{I_{i, j}}-g_{U_{i, j}}\right|^{2}
$$

for $\lambda \in \mathbb{R}$. A reader familiar with trace theorems for Sobolev spaces should consider our norm as a version of the trace norm of an Orlicz-Sobolev space corresponding to the Orlicz function $\psi(t)=t^{2} \log ^{\lambda}(e+t)$. The value $\lambda=-1$ is critical for Hölder continuous curves by the discussion above. Our first result implies that $1 / 2$-Hölder continuous Peano curves have finite energy $\mathcal{E}(g ; 2, \lambda)$ exactly when $\lambda<-1$.

Theorem 1.1. If $g: S^{1} \rightarrow \mathbb{R}^{2}$ is a Hölder continuous Peano curve then $\mathcal{E}(g ; 2,-1)=\infty$.

In order to deal with space-filling curves $g: S^{1} \rightarrow \mathbb{R}^{n}$, we modify the above definition by setting

$$
\mathcal{E}(g ; v, \lambda):=\sum_{i=1}^{\infty} i^{\lambda} \sum_{j=1}^{2^{i}}\left|g_{I_{i, j}}-g_{U_{i, j}}\right|^{\nu}
$$

for $v \in] 1, n]$ and $\lambda \in \mathbb{R}$. The space-filling analogue of Theorem 1.1 is

Theorem 1.2. If $g: S^{1} \rightarrow \mathbb{R}^{n}$ is a Hölder continuous space-filling curve then $\mathcal{E}(g ; n,-1)=\infty$.

Let us return to Peano curves in $W^{\frac{1}{2}, 2}\left(S^{1}\right)$. This energy that corresponds to $\mathcal{E}(g ; 2,0)$ in our notation is in principle much larger than $\mathcal{E}(g ; 2,-1)$, hence one expects a weaker 
optimal modulus of continuity than Hölder continuity. Indeed, in [7] the optimal modulus of continuity of general mappings $f \in W^{1,2}\left(B(0,1), \mathbb{R}^{n}\right)$ which guarantees that the 2-dimensional Hausdorff measure of $f(S)$ be zero, has been recognized to be

$$
|f(x)-f(y)| \leq C_{0} \exp \left(-C_{1} \log ^{1 / 2} \frac{2 \pi}{|x-y|}\right),
$$

where $C_{0}, C_{1}>0$ are any constants. To simplify our terminology, we use the term "spacefilling" even for Peano curves from now on. Our next results deal with general energies $\mathcal{E}(g ; n, \lambda)$.

Theorem 1.3. Let $g: S^{1} \rightarrow \mathbb{R}^{n}$ be a space-filling curve, satisfying

$$
|g(x)-g(y)| \leq C_{0} \exp \left(-C_{1} \log ^{\alpha} \frac{2 \pi}{|x-y|}\right)
$$

with some $\alpha \in] 0,1]$ and $C_{0}, C_{1}>0$, whenever $x, y \in S^{1}$ are close enough $\left(|x-y|<\epsilon_{0}\right.$ for some $\left.\epsilon_{0}>0\right)$. Then $\mathcal{E}(g ; n, \lambda)=\infty$ for $\lambda=n-1-\alpha n$, if $\alpha \geq \frac{n-1}{n}$, and for $\lambda>n-1-\alpha n$, if $\alpha<\frac{n-1}{n}$.

Note that, when $\alpha=1, g$ is assumed to be Hölder continuous.

Theorem 1.4. Let $g: S^{1} \rightarrow \mathbb{R}^{n}$ be a space-filling curve, satisfying

$$
|g(x)-g(y)| \leq C_{0} \log ^{-C_{1}} \frac{2 \pi}{|x-y|}
$$

with some $C_{0}, C_{1}>0$, whenever $x, y \in S^{1}$ are close enough. Then $\mathcal{E}(g ; n, n-1)=\infty$.

Note that by Theorem 3.1, the conclusion $\mathcal{E}(g ; v, \lambda)=\infty$ in Theorems 1.1-1.4 implies a weaker one that the integral in (7) is infinite. Also, $\mathcal{E}(g ; n, \lambda)=\infty$ for any space-filling curve $g: S^{1} \rightarrow \mathbb{R}^{n}$, when $\lambda>n-1$. This follows from the argument in [11].

In the process of verifying the above results, we actually prove a stronger statement, related to Hausdorff measures. It is given by the following theorem.

Theorem 1.5. Let $n \geq 2, v \in] 1, n], \lambda \in[-1, v-1]$ and $g: S^{1} \rightarrow \mathbb{R}^{n}$ be a continuous mapping with $\mathcal{E}(g ; v, \lambda)<\infty$, satisfying (4) with $\alpha=(v-1-\lambda) / v$, if $\lambda \in[-1,0]$, and $\alpha>(v-1-\lambda) / v$, if $\lambda \in] 0, v-1\left[\right.$. If $\lambda=v-1$, we assume that $g$ satisfies (5). Then $\mathcal{H}^{v}\left(g\left(S^{1}\right)\right)=0$.

Notice that $v$ is not necessarily an integer. Theorems $1.1-1.5$ are essentially sharp, see Sections 3 and 4 .

The proof of Theorem 1.5 is based on the following idea. We consider the balls $B\left(g_{i, j}, r_{i, j}\right)$ with

$$
g_{i, j}=g_{I_{i, j}} \text { and } r_{i, j}=\max \left\{\left|g_{I_{i, j}}-g_{U_{i, j}}\right|,\left|g_{I_{i, j}}-g_{I_{i+1, j}}\right|,\left|g_{I_{i, j}}-g_{I_{i+1, j}}\right|\right\},
$$

where $j_{1}$ and $j_{2}$ are such that $I_{i, j}=U_{i+1, j_{l}}, l=1,2$. Our energy assumption gives us control over a weighted double sum of $r_{i, j}^{v}$. Even though these balls cannot necessarily be used to cover $g\left(S^{1}\right)$, the given modulus of continuity allows us to construct a desired cover via this sequence of balls. For related arguments in the special case of quasiconformal mappings see $[10,9]$; also see $[7,11]$ for the setting of Sobolev mappings.

This paper is organized as follows. In the next section, we introduce our basic notation and give the proof of Theorem 1.5. We discuss the connection between our energy $\mathcal{E}(g ; v, \lambda)$ 
and the integral energy

$$
\int_{S^{1}} \int_{S^{1}} \frac{|g(x)-g(y)|^{v}}{|x-y|^{2}} \log ^{\lambda}\left(e+\frac{|g(x)-g(y)|}{|x-y|}\right) d \mathcal{H}_{x}^{1} d \mathcal{H}_{y}^{1}
$$

in Section 3. Finally, in Section 4, we construct an example, which shows the essential sharpness of our results.

\section{Proof of Theorem 1.5}

First, we would like to introduce our basic notation. Given a number $a>0$, we write $\lfloor a\rfloor$ for the largest integer less or equal to $a$. If $A$ is an arbitrary set, by $\sharp A$ we mean the total number of elements in $A$. By $\operatorname{diam}(A)$ and $\chi_{A}$, we denote the diameter and the characteristic function of the set $A \subset \mathbb{R}^{n}$, respectively. Given a point $x \in \mathbb{R}^{n}$ and a non-negative number $r$, $B(x, r)$ denotes an open ball centred in $x$ and having radius $r$. If $B$ is a ball, then $r(B)$ stands for its radius. We write $\mathcal{H}_{\delta}^{v}(A)$ with $v>0$ and $0<\delta \leq \infty$ for the $v$-dimensional Hausdorff content of a set $A$, while $\mathcal{H}^{v}(A)$ denotes its $v$-dimensional Hausdorff measure. If we write $L=L(\cdot)$, we mean that the number $L>0$ depends on the parameters listed in the parentheses. Finally, $C$ denotes a positive constant, which may depend on data and differ from occurrence to occurrence.

In addition to the standard Hausdorff content, we need a weighted Hausdorff content of a set $A \subset \mathbb{R}^{n}$ given by

$$
\lambda_{\infty}^{v}(A)=\inf \left\{\sum_{i=1}^{\infty} c_{i}\left(\operatorname{diam} E_{i}\right)^{v}: c_{i} \geq 0 \text { and } \chi_{A} \leq \sum_{i=1}^{\infty} c_{i} \chi_{E_{i}}\right\}
$$

for $v>0$. We refer to the collection of pairs $\left(E_{i}, c_{i}\right)_{i=1}^{\infty}$, such that $c_{i} \geq 0, E_{i} \subset \mathbb{R}^{n}$ and $\chi_{A} \leq \sum_{i=1}^{\infty} c_{i} \chi_{E_{i}}$, as a weighted covering of the set $A$. It is known that there exists a constant $\tau>0$, such that $\mathcal{H}_{\infty}^{v}(E) \leq \tau \lambda_{\infty}^{v}(E)$ for all bounded sets $E$ (see, for instance, [5], Corollary 8.2 and Theorem 9.7).

Before starting the proof, we state two simple lemmas concerning sequences.

Lemma 2.1. Let $\left\{a_{i}\right\}_{i \in \mathbb{N}}$ be an nondecreasing sequence of real numbers such that $1 \leq a_{i} \leq C_{0} i^{\beta}$ for each $i \geq i_{0}$ and some $C_{0}, \beta>0, i_{0} \in \mathbb{N}$. Then there exist constants $c=c(\beta)>0$ and $k_{0}=k_{0}\left(\beta, C_{0}, i_{0}\right) \in \mathbb{N}$, such that for any $k \geq k_{0}$, there are at least $6 k$ integers $i$ in the set $\{k, \ldots, 8 k\}$, satisfying

$$
c a_{i} \geq \frac{a_{i+1}-a_{i}}{\log i} i
$$

Proof. Let us fix an integer $k \geq i_{0}$ and assume that there exist numbers $\left\{i_{1}, \ldots, i_{k+1}\right\} \subset\{k, \ldots, 8 k\}$ such that

$$
c a_{i_{j}}<\frac{a_{i_{j}+1}-a_{i_{j}}}{\log i_{j}} i_{j}
$$

for $c=32 \beta$ and $j=1, \ldots, k+1$. Then

$$
\log a_{i_{j}+1}>\log a_{i_{j}}+\log \left(1+\frac{c \log i_{j}}{i_{j}}\right)>\log a_{i_{j}}+\frac{c \log i_{j}}{2 i_{j}}
$$


for all these $j$, provided $k>c \log k$. The monotonicity of $\left\{a_{i}\right\}$ and $\left\{\frac{\log i}{i}\right\}$ imply

$$
\log a_{8 k+1} \geq \log a_{k}+\frac{c}{2} \sum_{i=7 k}^{8 k} \frac{\log i}{i} \geq \frac{c \log 8 k}{16}
$$

On the other hand, $\log a_{8 k+1} \leq \log C_{0}+\beta \log (8 k+1)$, which is a contradiction when $k \geq k_{0}$, once $k_{0}=k_{0}\left(\beta, C_{0}, i_{0}\right)$ is fixed large enough.

Lemma 2.2. Let $\left\{a_{i}\right\}_{i \in \mathbb{N}}$ be a sequence of positive real numbers such that $a_{i} \leq C_{1} e^{C_{0} i}$ for each integer $i \geq i_{0}$ and some $C_{0}, C_{1}>0, i_{0} \in \mathbb{N}$. Then there exists a constant $c=c\left(C_{0}\right)>0$, such that there are infinitely many $i \in \mathbb{N}$ satisfying $c a_{i} \geq a_{i+1}-a_{i}$.

Proof. Trivial.

Proof of Theorem 1.5. The proof is somewhat different for non-positive values of $\lambda, \lambda \in] 0, v-1[$ and $\lambda=v-1$. We provide most of the details for all three cases. The origins of part of the proof for positive values of $\lambda$ can be found in [4], while the case of negative $\lambda$ adopts ideas of [7]. We may assume that the modulus of continuity (4) or (5) is satisfied globally, locality of the estimate will only influence the choices of parameters. The proof is broken into 5 steps.

Step 1. In this step we build our fundamental construction. In the end of the step, we decompose $S^{1}$ into smaller sets to be estimated separately.

Let $g_{i, j}$ and $r_{i, j}$ be as defined in (6) for $i=1,2, \ldots$ and $j=1, \ldots, 2^{i}$. Since the energy $\mathcal{E}(g ; v, \lambda)$ is finite, we have

$$
\sum_{i=1}^{\infty} \sum_{j=1}^{2^{i}} i^{\lambda} r_{i, j}^{v} \leq C \sum_{i=1}^{\infty} i^{\lambda} \sum_{j=1}^{2^{i}}\left|g_{I_{i, j}}-g_{U_{i, j}}\right|^{\nu}<\infty
$$

For each pair of indices $(i, j)$ with $r_{i, j}>0$, we define a collection of weighted balls $\mathcal{S}_{i, j}=$ $\left\{B_{i, j, k}=B\left(g_{i, j}, r_{i, j} / 2^{k}\right): k \in \mathbb{N}\right\}$, such that each ball $B=B_{i, j, k}$ has weight $w_{B}=v_{B} i^{\lambda}$, where $v_{B}=2^{k}$. We observe

$$
\sum_{B \in \mathcal{S}_{i, j}} w_{B} r^{v}(B)=i^{\lambda} r_{i, j}^{v} \sum_{k=1}^{\infty} \frac{1}{2^{k(v-1)}}=C_{v} i^{\lambda} r_{i, j}^{v}
$$

where $C_{v}=1 /\left(2^{v-1}-1\right)$. Defining $\mathcal{F}=\cup_{i, j} \mathcal{S}_{i, j}$, we have

$$
\sum_{B \in \mathcal{F}} w_{B} r^{v}(B) \leq C_{v} \sum_{i, j} i^{\lambda} r_{i, j}^{v}<\infty
$$

by (8). Some balls in $\mathcal{F}$ may coincide, however, we treat them as if they were different.

Pick $x \in S^{1}$ and a corresponding sequence of $\operatorname{arcs} I_{1}(x) \supset I_{2}(x) \supset \ldots$, such that $x \in I_{i}(x)$ and $I_{i}(x)=I_{i, j_{i}(x)}$ for some $j_{i}(x) \in\left\{1, \ldots, 2^{i}\right\}$. We denote $g_{i}(x)=g_{i, j_{i}(x)}$ and $r_{i}(x)=r_{i, j_{i}(x)}$. Then $g_{i}(x) \rightarrow g(x)$, when $i \rightarrow \infty$. Indeed, denoting by $\psi:[0, \infty[\rightarrow[0, \infty[$ the modulus of continuity of $g$, we obtain

$$
\left|g_{i}(x)-g(x)\right| \leq f_{I_{i, j_{i}(x)}}|g(y)-g(x)| d y \leq \int_{I_{i, j}(x)} \psi(|y-x|) d y \leq \psi\left(\operatorname{diam} I_{i, j_{i}(x)}\right) \leq \psi\left(\pi 2^{-i+1}\right) \rightarrow 0,
$$

when $i \rightarrow \infty$. Thus, we may assume existence of at least one pair of indices $(i, j)$ with $r_{i, j}>0$, otherwise $g\left(S^{1}\right)$ would be a one-point set. 
We neglect the set $\Gamma=\left\{x \in S^{1}: g_{i}(x)=g(x)\right.$ for all $\left.i \in \mathbb{N}\right\}$, whose image $g(\Gamma)$ is countable. Let $x \in S^{1} \backslash \Gamma$. We define the number $l_{0}(x) \in \mathbb{N}$ so that there are elements $g_{i}(x)$ for some $i \in \mathbb{N}$ outside the ball $B\left(g(x), 2^{-l_{0}(x)+1}\right)$, and construct a decomposition

$$
S^{1} \backslash \Gamma=\bigcup_{l \in \mathbb{N}} \Gamma_{l} \text {, where } \Gamma_{l}=\left\{x \in S^{1} \backslash \Gamma: l_{0}(x) \leq l\right\} .
$$

We denote $G_{l}=g\left(\Gamma_{l}\right)$, so that $g\left(S^{1} \backslash \Gamma\right)=\cup_{l \in \mathbb{N}} G_{l}$.

Step 2. Fix $l_{1} \in \mathbb{N}$. The remaining steps will be dedicated to the estimate for the set $\Gamma_{l_{1}}$. In this step, for each point $x \in \Gamma_{l_{1}}$ we define sequences that characterize how $g_{i}(x)$ converge to $g(x)$. We establish some important properties of these sequences, implied by the modulus of continuity, satisfied by $g$.

Let $x \in \Gamma_{l_{1}}$. For each integer $l \geq l_{1}$, let $a_{l}(x) \in \mathbb{N}$ be the smallest number, such that $g_{i}(x) \in B\left(x, 2^{-l+1}\right)$ for all $i>a_{l}(x)$. If $a_{l+1}(x)>a_{l}(x)$, we define $P_{l}(x)=\left\{a_{l}(x)+1, \ldots, a_{l+1}(x)\right\}$ and $p_{l}(x)=\sharp P_{l}(x)$, otherwise $P_{l}(x)=\left\{a_{l}(x)+1\right\}$ and $p_{l}(x)=0$.

Let $l \geq l_{1}$ and $i_{0}=a_{l+1}(x)$. In the case $\lambda \neq v-1$, we have

$$
2^{-l} \leq\left|g_{i_{0}}(x)-g(x)\right| \leq f_{I_{i_{0}}(x)}|g(y)-g(x)| d y \leq C_{0} \exp \left(-C_{1} \log ^{\alpha}\left(2^{i_{0}}\right)\right)=C_{0} \exp \left(-C i_{0}^{\alpha}\right),
$$

where $C=C_{1} \log ^{\alpha} 2$, because $|x-y| \leq 2 \pi / 2^{i}$, when $x, y \in I_{i}(x)$. The last estimate implies $a_{l+1}(x)+1=i_{0}+1 \leq c_{0} l^{1 / \alpha}$, thus $i \leq c_{0} l^{1 / \alpha}$ for each $i \in P_{l}(x)$, with some constant $c_{0}=$ $c_{0}\left(\alpha, C_{0}, C_{1}\right)>0$. Similarly, when $\lambda=v-1$, we obtain $a_{l}(x) \leq c_{1} e^{c_{0} l}$ for each $l \geq l_{1}$, with $c_{1}=c_{1}\left(C_{0}, C_{1}\right)>0$ and $c_{0}=c_{0}\left(C_{1}\right)>0$.

If $\lambda \neq v-1$, we denote

$$
\theta_{l}(x)= \begin{cases}1, & \text { if } p_{l}(x) \leq c l^{\frac{1-\alpha}{\alpha}}, \\ 0, & \text { otherwise, }\end{cases}
$$

for $l \geq l_{1}$ and a constant $c \in \mathbb{N}$, which we will specify later. We use ideas originating from $[9,14]$ in order to prove that there exists an integer $l^{\prime} \geq 2 l_{1}$, such that

$$
\sum_{k=l_{1}}^{l} \theta_{k}(x) \geq \frac{l}{2}
$$

for each $l \geq l^{\prime}$. In other words, at least half of the annuli do not contain too many centres from $\left(g_{i}(x)\right)_{i \in \mathbb{N}}$.

Let us assume that (10) does not hold for some $l \geq 2 l_{1}$. We obtain a lower bound for $a_{l+1}(x)$, using the assumption that we have at least $\lfloor l / 2\rfloor-l_{1}+2$ integers $k \in\left\{l_{1}, \ldots, l\right\}$ with $\theta_{k}(x)=0$. We have

$$
c_{0} l^{1 / \alpha} \geq a_{l+1}(x) \geq \sum_{\substack{k=l_{1}, \ldots, l \\ \theta_{k}(x)=0}} p_{k}(x) \geq \sum_{k=l_{1}}^{\lfloor l / 2\rfloor+1} c k^{\frac{1-\alpha}{\alpha}} \geq c \alpha 2^{-\frac{1}{\alpha}} l^{\frac{1}{\alpha}}-c \alpha l_{1}^{\frac{1}{\alpha}}
$$

which can not be true, if $c$ is fixed so that $c>c_{0} 2^{1 / \alpha} / \alpha$, and $l$ is large enough. In other words, there exists a number $l^{\prime}=l^{\prime}\left(l_{1}, \alpha, C_{0}, C_{1}\right) \in \mathbb{N}$, such that (10) holds for all $l \geq l^{\prime}$. For completeness, in the case $\lambda=v-1$, we put $\theta_{l}(x)=1$ for each $l \geq l_{1}$. 
Step 3. In the following two steps, we prove that for a substantial number of integers $k$, we may find a set of balls $B \in \mathcal{F}$ with radii in a suitable range and centres in $B\left(g(x), 2^{-k+1}\right)$, such that $\sum r^{v}(B) w_{B} \geq$ const $\cdot 2^{-v k} k^{-1+\delta}$ for some $\delta \geq 0$. When $\lambda \neq v-1, \delta$ is chosen so that $\alpha=(v-1-\lambda) /(v-2 \delta)$; otherwise, $\delta=1$. Note that $\delta=0$, when $\lambda \leq 0$. Without the loss of generality, we may assume that $\delta \leq 1$. In this step, we define the desired collection of balls and provide the estimates for $\sum r^{v}(B) v_{B}$.

First, we concentrate on the case $\lambda>0$. Let us take $k \geq l_{1}$ with $\theta_{k}(x)=1$. We find a set of balls $M_{k}(x) \subset \mathcal{F}$ with radii in the range $\left[2^{-k-3} / \sharp P_{k}(x), 2^{-k-2} / \sharp P_{k}(x)\right.$, satisfying

$$
\sum_{B \in M_{k}(x)} r^{v}(B) v_{B} \geq 2^{-v k-3 v+1}\left(\sharp P_{k}(x)\right)^{1-v} .
$$

By the definitions of $P_{k}(x)$ and $r_{i}(x)$, we have

$$
\sum_{i \in P_{k}(x)} r_{i}(x) \geq \frac{2^{-k}}{2}
$$

Therefore,

$$
\sum_{\substack{i \in P_{k}(x) \\ P^{-k-2} / \sharp P_{k}(x)}} r_{i}(x) \geq \frac{2^{-k}}{4}
$$

For each $i \in P_{k}(x)$, such that $r_{i}(x) \geq 2^{-k-2} / \sharp P_{k}(x)$, we define a number $q_{k, i}(x) \in \mathbb{N}$, satisfying

$$
\frac{2^{-k+q_{k, i}(x)-1}}{4 \cdot \sharp P_{k}(x)} \leq r_{i}(x)<\frac{2^{-k+q_{k, i}(x)}}{4 \cdot \sharp P_{k}(x)} .
$$

Then the collection $M_{k}(x)$ is defined by

$$
M_{k}(x)=\left\{B_{i, j, q}: i \in P_{k}(x), r_{i}(x) \geq 2^{-k-2} / \sharp P_{k}(x), j=j_{i}(x), q=q_{k, i}(x)\right\} .
$$

We have

$$
\frac{2^{-k}}{8 \cdot \sharp P_{k}(x)} \leq r(B)<\frac{2^{-k}}{4 \cdot \sharp P_{k}(x)} \leq \frac{2^{-k}}{4}
$$

for each $B \in M_{k}(x)$, which gives

$$
\frac{2^{-k}}{8 c k^{(1-\alpha) / \alpha}} \leq r(B)<\frac{2^{-k}}{4}
$$

for each $B \in M_{k}(x)$, when $\left.\lambda \in\right] 0, v-1$. Additionally,

$$
\sum_{B \in M_{k}(x)} r(B) v_{B}=\sum_{\substack{i \in P_{k}(x) \\ r_{i}(x) \geq 2^{-k-2} / \sharp P_{k}(x)}} r_{i}(x) \geq \frac{2^{-k}}{4} .
$$

Finally,

$$
\sum_{B \in M_{k}(x)} r^{v}(B) v_{B} \geq\left(\frac{2^{-k}}{8 \cdot \sharp P_{k}(x)}\right)^{v-1} \sum_{B \in M_{k}(x)} r(B) v_{B} \geq \frac{2^{-v k}}{2^{3 v-1}\left(\sharp P_{k}(x)\right)^{v-1}},
$$

as desired. Note also that this argument works even if $p_{k}(x)=0$, because in that case the set $P_{k}(x)$ consists of one index $i$ with $g_{i}(x) \in B\left(g(x), 2^{-k}\right)$. The definition of $r_{i, j}$ implies $r_{i}(x)>2^{-k}$.

For non-positive $\lambda$, similarly, we take a number $k \geq l_{1}$ with $\theta_{k}(x)=1$. Starting with (12), we proceed a similar proof with $\sharp P_{k}(x)$ replaced by $c k^{\frac{1-\alpha}{\alpha}}$ (note the case $p_{k}(x)=0$ ). 
This gives us a set of balls $M_{k}(x) \subset \mathcal{F}$ centred in $B\left(g(x), 2^{-k+1}\right)$ with radii in the range $\left[c^{-1} 2^{-k-3} k^{(\alpha-1) / \alpha}, c^{-1} 2^{-k-2} k^{(\alpha-1) / \alpha}[\right.$, and satisfying

$$
\sum_{B \in M_{k}(x)} r^{v}(B) v_{B} \geq c^{1-v} 2^{-v k-3 v+1} k^{(v-1)(\alpha-1) / \alpha}
$$

When $\theta_{k}(x)=0$, for completeness, we put $M_{k}(x)=\emptyset$.

Step 4. In this step we complete the estimates, announced in the beginning of the previous step, by estimating the index $i$ of each ball $B_{i, j, q} \in M_{k}(x)$.

Let us consider $\lambda \in] 0, v-1$ [ only. We apply Lemma 2.1 to $\beta=1 / \alpha, C_{0}=c_{0}$ and $i_{0}=l^{\prime}$, obtaining an integer $k_{1}=k_{1}\left(\alpha, c_{0}, l^{\prime}\right)$ and a constant $c_{1}=c_{1}(\alpha)>0$, such that for each $k_{0} \geq k_{1}$ the set $\left\{k_{0}, \ldots, 8 k_{0}\right\}$ contains at least $6 k_{0}$ numbers $k$ with the property

$$
i>a_{k}(x)>\frac{c_{1}\left(a_{k+1}(x)-a_{k}(x)\right) k}{\log k}=\frac{c_{1} p_{k}(x) k}{\log k},
$$

whenever $i \in P_{k}(x)$. Clearly, we may also assume that $\log ^{\lambda} k<k^{\delta}$ for all $k>k_{1}$. Fix $k_{0} \geq k_{1}$. By (10), we have at most $4 k_{0}$ integers $k$ from the set $\left\{k_{0}, \ldots, 8 k_{0}\right\}$, such that $p_{k}(x)>c k^{(1-\alpha) / \alpha}$. Together with (11), all these facts imply that, for more than $k_{0}$ integers $k$ in the set $\left\{k_{0}, \ldots, 8 k_{0}\right\}$, we have either $p_{k}(x) \neq 0$ and

$$
\sum_{B \in M_{k}(x)} r^{v}(B) w_{B} \geq C 2^{-v k} \frac{k^{\lambda}}{\log ^{\lambda} k} p_{k}^{\lambda-v+1}(x) \geq C 2^{-v k} k^{\lambda-(v-1-\lambda)(1-\alpha) / \alpha-\delta}=\tilde{C} 2^{-v k} k^{-1+\delta} ;
$$

or $p_{k}(x)=0$ and

$$
r^{v}(B) w_{B} \geq r^{v}(B) v_{B} \geq \tilde{C} 2^{-v k} \geq \tilde{C} 2^{-v k} k^{-1+\delta}
$$

for the only ball $B$ in $M_{k}(x)$. Here $\tilde{C}$ is some positive constant, which depends on $v, C_{0}, C_{1}, \alpha$ and $\lambda$.

Let us obtain an analogue of this for $\lambda \in[-1,0]$. For each $k \geq l_{1}$ with $\theta_{k}(x)=1$ we observe by (14) and $a_{k+1}(x)+1 \leq c_{0} k^{1 / \alpha}$ that

$$
\sum_{B \in M_{k}(x)} r^{v}(B) w_{B} \geq C 2^{-v k} k^{[(\alpha-1)(v-1)+\lambda] / \alpha}=\tilde{C} 2^{-v k} k^{-1},
$$

where we may have redefined the value of $\tilde{C}$, but it still depends on $v, \alpha, \lambda, C_{0}$ and $C_{1}$ only. By (10), we obtain more than $k_{0}$ integers $k \in\left\{k_{0}, \ldots, 8 k_{0}\right\}$ with the required property.

Finally, in the case $\lambda=v-1$, we apply Lemma 2.2. There exists $k \geq k_{0}$, such that $i>a_{k}(x) \geq C\left(a_{k+1}(x)-a_{k}(x)\right)=C p_{k}(x)$, whenever $i \in P_{k}(x)$. Combining this with (11), we obtain

$$
\sum_{B \in M_{k}(x)} r^{v}(B) w_{B} \geq C 2^{-v k}
$$

for this $k$ (note $p_{k}(x)=0$ again).

Step 5. In this step we complete the estimates for $\Gamma_{l_{1}}$ and the proof.

We return to the case $\lambda \neq v-1$. For $k \in\left\{k_{0}, \ldots, 8 k_{0}\right\}$, let us define

$$
S_{k}=\left\{x \in \Gamma_{l_{1}}: \sum_{B \in M_{k}(x)} r^{v}(B) w_{B} \geq \tilde{C} 2^{-v k} k^{-1+\delta}\right\} \text { and } \tilde{M}_{k}=\bigcup_{x \in S_{k}} M_{k}(x) .
$$


The previous argument implies

$$
\sum_{k=k_{0}}^{8 k_{0}} \chi_{g\left(S_{k}\right)} \geq k_{0} \chi_{G_{l_{1}}}
$$

We finish the proof for $\lambda \in] 0, v-1[$. The techniques used are the ones applied in [4] in cases " $\mathrm{C}$ " and "D". If $k_{1}, k_{2} \in\left\{k_{0}, \ldots, 8 k_{0}\right\}$ are such that $k_{1} \geq k_{2}+1+\log _{2} c+\frac{1-\alpha}{\alpha} \log _{2} k_{2}$, then

$$
\frac{2^{-k_{1}}}{4} \leq \frac{2^{-k_{2}}}{8 c k_{2}^{(1-\alpha) / \alpha}}
$$

thus, $\tilde{M}_{k_{1}} \cap \tilde{M}_{k_{2}}=\emptyset$ by (13). This means that there exists a constant $C^{\prime}$, independent of $k_{0}$, such that $\tilde{M}_{k_{1}} \cap \tilde{M}_{k_{2}}=\emptyset$ for each $k_{1}, k_{2} \in\left\{k_{0}, \ldots, 8 k_{0}\right\}$ with $k_{1}-k_{2} \geq\left\lfloor C^{\prime} \log k_{0}\right\rfloor \geq 1$, once $k_{0}$ is large enough. We prove next that there are not so many numbers $k \in\left\{k_{0}, \ldots, 8 k_{0}\right\}$, satisfying (16). Let us assume that there are at least $\left\lfloor k_{0} / 2\right\rfloor$ collections $\tilde{M}_{k}, k \in\left\{k_{0}, \ldots, 8 k_{0}\right\}$, with

$$
\sum_{B \in \tilde{M}_{k}} r^{v}(B) w_{B}>k^{\delta / 2-1} \text {. }
$$

The previous conclusion implies that there are at least $\frac{\left\lfloor k_{0} / 2\right\rfloor}{\left\lfloor C^{\prime} \log k_{0}\right\rfloor}$ collections $\tilde{M}_{k}$ with the property (16), consisting of different balls each. Therefore, we obtain

$$
\sum_{B \in \mathcal{F}} r^{v}(B) w_{B} \geq \frac{\left\lfloor k_{0} / 2\right\rfloor}{\left\lfloor C^{\prime} \log k_{0}\right\rfloor} 8^{\delta / 2-1} k_{0}^{\delta / 2-1}
$$

which is a contradiction with (9), once $k_{0} \geq 2$ is large enough. Therefore, there are at most $\left\lfloor k_{0} / 2\right\rfloor-1$ collections $\tilde{M}_{k}$, satisfying (16), which yields

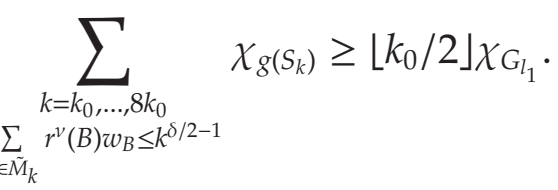

Let us pick $k \in\left\{k_{0}, \ldots, 8 k_{0}\right\}$ with $S_{k} \neq \emptyset$, such that (16) is not true. By the Besicovitch covering theorem, we cover $g\left(S_{k}\right)$ with a finite collection of balls $\left\{B_{k, i}=B\left(g\left(x_{k, i}\right), 2^{-k+1}\right)\right\}_{i \in I_{k}}$ with $x_{k, i} \in S_{k}$, such that $\sum_{i} \chi_{B_{k, i}}(y) \leq N$ for each $y \in \mathbb{R}^{n}$ and some $N=N(n) \in \mathbb{N}$. We observe by the definition of $S_{k}$

$$
\sharp I_{k} \cdot 2^{-v k+2 v} \leq 4^{v} \frac{k^{1-\delta}}{\tilde{C}} \sum_{i \in I_{k}} \sum_{B \in M_{k}\left(x_{k, i}\right)} r^{v}(B) w_{B} \leq \frac{4^{v} N}{\tilde{C}} k^{1-\delta} \sum_{B \in \tilde{M}_{k}} r^{v}(B) w_{B} \leq \frac{4^{v} N}{\tilde{C}} k^{-\delta / 2},
$$

where we have used the fact that all balls in $M_{k}\left(x_{k, i}\right)$, with $i \in I_{k}$, are centred in $B_{k, i}$. Since $\bigcup_{k}\left\{\left(B_{k, i},\left\lfloor k_{0} / 2\right\rfloor^{-1}\right): i \in I_{k}\right\}$ is a weighted covering of the set $G_{l_{1}}$, we obtain $\left(I_{k}=\emptyset\right.$, if $\left.S_{k}=\emptyset\right)$

$$
\begin{aligned}
\mathcal{H}_{\infty}^{v}\left(G_{l_{1}}\right) \leq \tau \lambda_{\infty}^{v}\left(G_{l_{1}}\right) \leq \frac{\tau}{\left\lfloor k_{0} / 2\right\rfloor} \sum_{\substack{\sum_{\begin{subarray}{c}{k=M_{0}, \ldots, 8 k_{0} \\
B \in M_{k}} }} r^{v}(B) w_{B} \leq k^{\delta / 2-1}}\end{subarray}} \sharp I_{k} \cdot 2^{-v k+2 v} & \leq \frac{4^{v} N \tau}{\tilde{C}\left\lfloor k_{0} / 2\right\rfloor} \sum_{k=k_{0}}^{8 k_{0}} k^{-\delta / 2} \\
& \leq \frac{2^{2 v+5} N \tau}{\tilde{C}} k_{0}^{-\delta / 2} .
\end{aligned}
$$

Finally, letting $k_{0}$ go to infinity, we obtain $\mathcal{H}_{\infty}^{v}\left(G_{l_{1}}\right)=0$. This implies $\mathcal{H}^{v}\left(G_{l_{1}}\right)=0$ and $\mathcal{H}^{v}\left(g\left(S^{1} \backslash \Gamma\right)\right)=0$. 
In the case of non-positive $\lambda$, the proof is more trivial. We simply notice that $\tilde{M}_{k_{1}} \cap \tilde{M}_{k_{2}}=\emptyset$ for each $k_{1} \neq k_{2}$. As in (17), using the Besicovitch covering theorem and the definition of $S_{k}$, we find a covering $\left\{B_{k, i}\right\}_{i \in I_{k}}$ of the set $g\left(S_{k}\right)$, such that

$$
\sum_{i \in I_{k}} \operatorname{diam}^{v}\left(B_{k, i}\right)=\sharp I_{k} \cdot 2^{-v k+2 v} \leq \frac{4^{v} N}{\tilde{C}} k \sum_{B \in \tilde{M}_{k}} r^{v}(B) w_{B} .
$$

We conclude

$$
\mathcal{H}_{\infty}^{v}\left(G_{l_{1}}\right) \leq \frac{\tau}{k_{0}} \sum_{k=k_{0}}^{8 k_{0}} \sharp I_{k} \cdot 2^{-v k+2 v} \leq \frac{4^{v} N \tau}{\tilde{C} k_{0}} \sum_{k=k_{0}}^{8 k_{0}} k \sum_{B \in \tilde{M}_{k}} r^{v}(B) w_{B} \leq \frac{2^{2 v+3} N \tau}{\tilde{C}} \sum_{\substack{B \in \mathcal{F} \\ r(B) \leq 2^{-k_{0}}}} r^{v}(B) w_{B} \rightarrow 0,
$$

when $k_{0} \rightarrow \infty$.

Finally, in the case $\lambda=v-1$, we apply the Besicovitch covering theorem to obtain a collection of balls $\left\{B_{i}=B\left(g\left(x_{i}\right), 2^{-k_{i}+1}\right)\right\}_{i \in I}$ with $x_{i} \in \Gamma_{l_{1}}$ and $k_{i} \geq k_{0}$, covering the set $G_{l_{1}}$, such that $\sum_{i} \chi_{B_{i}}(y) \leq N$ and (15) holds with $x=x_{i}$ and $k=k_{i}$ for each $i \in I$. We estimate

$$
\mathcal{H}_{\infty}^{v}\left(G_{l_{1}}\right) \leq \sum_{i \in I} 2^{-v k_{i}+2 v} \leq C \sum_{i \in I} \sum_{B \in M_{k}\left(x_{i}\right)} r^{v}(B) w_{B} \leq C N \sum_{\substack{B \in \mathcal{F} \\ r(B) \leq 2^{-k_{0}}}} r^{v}(B) w_{B} \rightarrow 0,
$$

when $k_{0} \rightarrow \infty$.

\section{How to COMPUTE THE ENERGY}

In this section, we use a fixed dyadic decomposition of $S^{1}$ again. We denote by $\mathcal{D}_{i}$ the set of $2^{i}$ dyadic intervals of $S^{1}$ of generation $i$, having length $2^{1-i} \pi$. In this section, we denote the parent interval of $I \in \mathcal{D}_{i}$ by $U(I) \in \mathcal{D}_{i-1}$. Recall that, the parent interval of $I$ is the dyadic interval of previous generation which contains $I$.

Theorem 3.1. Let $g: S^{1} \rightarrow \mathbb{R}^{n}, n \geq 2$, be continuous. Let $\lambda \in \mathbb{R}$ and $v>1$. If

$$
\int_{S^{1}} \int_{S^{1}} \frac{|g(x)-g(y)|^{v}}{|x-y|^{2}} \log ^{\lambda}\left(e+\frac{|g(x)-g(y)|}{|x-y|}\right) d \mathcal{H}_{x}^{1} d \mathcal{H}_{y}^{1}<\infty,
$$

then $\mathcal{E}(g ; v, \lambda)<\infty$.

Proof. We may assume that $|g(x)| \leq 1$ for all $x \in S^{1}$. Let

$$
A_{k}(y)=\left\{x \in S^{1}: 2^{-k+1} \pi<|x-y|_{S^{1}} \leq 2^{-k+2} \pi\right\}
$$

for each $k \in \mathbb{N}$, where $|\cdot|_{S^{1}}$ is the length of the shorter arc of $S^{1}$ connecting $x$ and $y$. In the following we will use the notation

$$
\Delta g(x, y):=\frac{|g(x)-g(y)|}{|x-y|}
$$

and

$$
A_{I, k}:=\int_{I} f_{A_{k}(y)} \Delta g(x, y) d \mathcal{H}_{x}^{1} d \mathcal{H}_{y}^{1},
$$

where $I$ is some dyadic interval and $k \in \mathbb{N}$. Here and in what follows, we use the convention that $f_{A_{1}(y)} \psi(x, y) d \mathcal{H}_{x}^{1}=0$ for all $y \in S^{1}$ and any function $\psi: S^{1} \times S^{1} \rightarrow \mathbb{R}$. 
Let $\varphi:[0, \infty[\rightarrow[0, \infty[$ and $\tilde{\varphi}:[0, \infty[\rightarrow[0, \infty[$ be two increasing continuous functions such that $\varphi$ is convex on $\left[0, \infty\left[\right.\right.$ and $\varphi(t)=t^{v} \log ^{\lambda}(e+t), \tilde{\varphi}(y)=y^{\frac{1}{v}} \log ^{-\lambda / v}(e+y)$, for $t>t_{0}$ and $y>\varphi\left(t_{0}\right)$, where $t_{0}$ is a suitably fixed number. In addition, we require that $\tilde{\varphi}(\varphi(t)) \geq C t$ for all $t \geq t_{0}$ and some constant $C>0$. Let $I \in \mathcal{D}_{i}$. Using these facts and Jensen's inequality we obtain

$$
\text { (18) } \begin{aligned}
& f_{I} f_{U(I)}|g(x)-g(y)| d \mathcal{H}_{x}^{1} d \mathcal{H}_{y}^{1} \leq C \sum_{k=i}^{\infty} 2^{i-2 k} f_{I} f_{A_{k}(y)} \Delta g(x, y) d \mathcal{H}_{x}^{1} d \mathcal{H}_{y}^{1} \\
& =C \sum_{\substack{k=i \\
A_{I, k} \leq t_{0}}}^{\infty} 2^{i-2 k} f_{I} f_{A_{k}(y)} \Delta g(x, y) d \mathcal{H}_{x}^{1} d \mathcal{H}_{y}^{1}+C \sum_{\substack{k=i \\
A_{I, k}>t_{0}}}^{\infty} 2^{i-2 k} f_{I} f_{A_{k}(y)} \Delta g(x, y) d \mathcal{H}_{x}^{1} d \mathcal{H}_{y}^{1} \\
& \leq C t_{0} \sum_{k=i}^{\infty} 2^{i-2 k}+C \sum_{\substack{k=i \\
A_{I, k}>t_{0}}}^{\infty} 2^{i-2 k} \tilde{\varphi}\left(\varphi\left(f_{I} f_{A_{k}(y)} \Delta g(x, y) d \mathcal{H}_{x}^{1} d \mathcal{H}_{y}^{1}\right)\right) \\
& \leq C 2^{-i}+C \sum_{\substack{k=i \\
A_{l, k} t_{0}}} 2^{i-2 k} \tilde{\varphi}\left(f_{I} f_{A_{k}(y)} \varphi(\Delta g(x, y)) d \mathcal{H}_{x}^{1} d \mathcal{H}_{y}^{1}\right) \\
& \leq C \sum_{k=i}^{\infty} 2^{i-2 k}\left(f_{I} f_{A_{k}(y)} \varphi(\Delta g(x, y)) d \mathcal{H}_{x}^{1} d \mathcal{H}_{y}^{1}\right)^{1 / v} \log ^{-\lambda / v}\left(e+f_{I} f_{A_{k}(y)} \varphi(\Delta g(x, y)) d \mathcal{H}_{x}^{1} d \mathcal{H}_{y}^{1}\right) \\
& +C 2^{-i} .
\end{aligned}
$$

Raising the last term on the RHS of (18) to the power of $v$, multiplying it by $i^{\lambda}$ and summing over all $I \in \mathcal{D}_{i}$ and over all $i \in \mathbb{N}$, we get some finite number. Now we concentrate on the first term on the RHS of (18). For simplicity we denote

$$
V_{I, k}:=\int_{I} f_{A_{k}(y)} \varphi(\Delta g(x, y)) d \mathcal{H}_{x}^{1} d \mathcal{H}_{y}^{1}
$$

in the following. We start with Hölder's inequality:

$$
\begin{aligned}
& 2^{i} \sum_{k=i}^{\infty} 2^{-2 k}\left(V_{I, k}\right)^{1 / v} \log ^{-\lambda / v}\left(e+V_{I, k}\right) \leq 2^{i}\left[\sum_{k=i}^{\infty} 2^{-k \frac{v}{v-1}}\right]^{(v-1) / v}\left[\sum_{k=i}^{\infty} 2^{-v k} V_{I, k} \log ^{-\lambda}\left(e+V_{I, k}\right)\right]^{1 / v} \\
& \leq C\left[\sum_{k=i}^{\infty} 2^{-v k} V_{I, k} \log ^{-\lambda}\left(e+V_{I, k}\right)\right]^{1 / v} .
\end{aligned}
$$

From here on we split the argument into two cases according to the value of $\lambda$. Assume first that $\lambda \leq 0$. Since $|g| \leq 1$ and $\varphi(t) \leq \varphi\left(t_{0}\right)+t^{v} \log ^{\lambda}(e+t)$, we have, for $k \geq 1$,

$$
e+f_{I} f_{A_{k}(y)} \varphi\left(\frac{|g(x)-g(y)|}{|x-y|}\right) d x d y \leq e+\varphi\left(t_{0}\right)+2^{v k} \leq 2^{\left(v+C_{1}\right) k},
$$

where $C_{1}$ depends on $\varphi\left(t_{0}\right)$ and $v$ only. Therefore,

$$
\log ^{-\lambda}\left(e+V_{11}\right) \leq C k^{-\lambda}
$$


We estimate now the contribution of the first term on the right hand side of (18) to the sum (2). Using estimates (18), (19) and (20) and the definitions of $I$ and $A_{k}(y)$ we obtain the estimate

$$
\begin{aligned}
& \sum_{i=1}^{\infty} \sum_{I \in \mathcal{D}_{i}}\left(2^{i} \sum_{k=i}^{\infty} 2^{-2 k}\left(V_{I, k}\right)^{1 / v} \log ^{-\lambda / v}\left(e+V_{I, k}\right)\right)^{v} i^{\lambda} \\
& \leq C \sum_{i=1}^{\infty} \sum_{I \in \mathcal{D}_{i}} \sum_{k=i}^{\infty} 2^{-v k} i^{\lambda} V_{I, k} \log ^{-\lambda}\left(e+V_{I, k}\right) \\
& \leq C \sum_{k=1}^{\infty} \sum_{i=1}^{k} \sum_{I \in \mathcal{D}_{i}} 2^{-v k} i^{\lambda} k^{-\lambda} f_{I} f_{A_{k}(y)} \varphi(\Delta g(x, y)) d x d y \\
& \leq C \sum_{k=1}^{\infty} \sum_{i=1}^{k} 2^{i} i^{\lambda} 2^{-k} k^{-\lambda} \sum_{I \in \mathcal{D}_{i}} \int_{I} \int_{A_{k}(y)} \frac{|g(x)-g(y)|^{v}}{|x-y|^{2}} \log ^{\lambda}\left(e+\frac{|g(x)-g(y)|}{|x-y|}\right) d \mathcal{H}_{x}^{1} d \mathcal{H}_{y}^{1} \\
& +C \sum_{k=1}^{\infty} \sum_{i=1}^{k} \sum_{I \in \mathcal{D}_{i}} 2^{-v k} i^{\lambda} k^{-\lambda} \varphi\left(t_{0}\right) \\
& \leq C \sum_{k=1}^{\infty} \underbrace{\left[\sum_{i=1}^{k} 2^{i} i^{\lambda}\right]}_{\approx 2^{k} k^{\lambda}} 2^{-k} k^{-\lambda} \int_{S^{1}} \int_{A_{k}(y)} \frac{|g(x)-g(y)|^{v}}{|x-y|^{2}} \log ^{\lambda}\left(e+\frac{|g(x)-g(y)|}{|x-y|}\right) d \mathcal{H}_{x}^{1} d \mathcal{H}_{y}^{1} \\
& +C \sum_{k=1}^{\infty} 2^{-(v-1) k} \varphi\left(t_{0}\right) \\
& \leq C \sum_{k=1}^{\infty} \int_{S^{1}} \int_{A_{k}(y)} \frac{|g(x)-g(y)|^{v}}{|x-y|^{2}} \log ^{\lambda}\left(e+\frac{|g(x)-g(y)|}{|x-y|}\right) d \mathcal{H}_{x}^{1} d \mathcal{H}_{y}^{1}+C \\
& =C \int_{S^{1}} \int_{S^{1}} \frac{|g(x)-g(y)|^{\nu}}{|x-y|^{2}} \log ^{\lambda}\left(e+\frac{|g(x)-g(y)|}{|x-y|}\right) d \mathcal{H}_{x}^{1} d \mathcal{H}_{y}^{1}+C<\infty .
\end{aligned}
$$

This finishes the proof in the case $\lambda \leq 0$.

We may now assume that $\lambda>0$. In order to estimate the logarithmic term from above, we define

$$
\chi(I, k)= \begin{cases}1, & \text { if } V_{I, k} \geq 2^{k \frac{\nu-1}{2}} \\ 0, & \text { otherwise. }\end{cases}
$$


We start with the estimate (19) as in previous calculation to obtain the estimate

$$
\begin{aligned}
& \sum_{i=1}^{\infty} \sum_{I \in \mathcal{D}_{i}}\left(2^{i} \sum_{k=i}^{\infty} 2^{-2 k}\left(V_{I, k}\right)^{1 / v} \log ^{-\lambda / v}\left(e+V_{I, k}\right)\right)^{v} i^{\lambda} \\
& \leq C \sum_{k=1}^{\infty} \sum_{i=1}^{k} \sum_{I \in \mathcal{D}_{i}} 2^{-v k} i^{\lambda} V_{I, k} \log ^{-\lambda}\left(e+V_{I, k}\right) \\
& \leq C \sum_{k=1}^{\infty} \sum_{i=1}^{k} \sum_{I \in \mathcal{D}_{i}} \chi(I, k) 2^{-v k} i^{\lambda} V_{I, k} \log ^{-\lambda}\left(e+V_{I, k}\right) \\
& \quad+C \sum_{k=1}^{\infty} \sum_{i=1}^{k} \sum_{I \in \mathcal{D}_{i}}(1-\chi(I, k)) 2^{-v k} i^{\lambda} V_{I, k} \log ^{-\lambda}\left(e+V_{I, k}\right)=: P_{1}+P_{2} .
\end{aligned}
$$

In order to estimate $P_{1}$ we may proceed in the same way as in the case $\lambda \leq 0$. For $P_{2}$ we use the definition of $\chi$ as follows

$$
P_{2} \leq C \sum_{k=1}^{\infty} \sum_{i=1}^{k} \sum_{I \in \mathcal{D}_{i}}(1-\chi(I, k)) 2^{-v k} 2^{k \frac{v-1}{2}} i^{\lambda} \leq C \sum_{k=1}^{\infty} 2^{-v k} 2^{k \frac{v-1}{2}} \sum_{i=1}^{k} 2^{i} i^{\lambda} \leq C \sum_{k=1}^{\infty} 2^{-k \frac{v-1}{2}} k^{\lambda} \leq C .
$$

Therefore, we obtain

$$
P_{1}+P_{2} \leq C \int_{S^{1}} \int_{S^{1}} \frac{|g(x)-g(y)|^{v}}{|x-y|^{2}} \log ^{\lambda}\left(e+\frac{|g(x)-g(y)|}{|x-y|}\right) d \mathcal{H}_{x}^{1} d \mathcal{H}_{y}^{1}+C
$$

\section{EXAMPLE}

This section is dedicated to the following example. Note that $v$ is not necessarily an integer.

Example 1. Let $n \geq 2,1<v \leq n$ and $\lambda \in[-1, v-1]$. If $\lambda \neq v-1$, assume $0<1 / \beta<\frac{v-1-\lambda}{v}$; otherwise, take $\beta>\frac{v}{v-1}$. There exists a mapping $f: S^{1} \rightarrow \mathbb{R}^{n}$ with

$$
\int_{S^{1}} \int_{S^{1}} \frac{|f(x)-f(y)|^{\nu}}{|x-y|^{2}} \log ^{\lambda}\left(e+\frac{|f(x)-f(y)|}{|x-y|}\right) d \mathcal{H}_{x}^{1} d \mathcal{H}_{y}^{1}<\infty
$$

and $\mathcal{H}^{v}\left(f\left(S^{1}\right)\right)>0$, which is continuous with modulus of continuity $\varphi(t)=$ $C_{0} \exp \left(-C_{1} \log ^{1 / \beta} \frac{1}{t}\right)$, if $\lambda \neq v-1$, and $\varphi(t)=C_{0} \exp \left(-C_{1} \log ^{\frac{1}{\beta}} \log \frac{1}{t}\right)$, otherwise; where $C_{0}>0$ and $C_{1}>0$ depend on the given parameters.

In the following subsections, we concentrate on the case $\lambda \neq v-1$. The remaining case is addressed in Subsection 4.9 in the end of this section.

4.1. Construction. The following construction is a modification to the example given in [8]. We will define the map $f: I \rightarrow \mathbb{R}^{n}$, where $I \in \mathbb{R}$ is an interval. It is easy to modify the construction to a map $f: S^{1} \rightarrow \mathbb{R}^{n}$.

Put $R_{j}=\exp \left(-j^{\beta}\right)$ and $r_{j}=2^{n} \exp \left(-(j+1)^{\beta}\right)$. Let $n_{0}$ be a large fixed integer, which will be determined later. We utilize the numbers $R_{j}$ and $r_{j}$, with $j \geq n_{0}$, in a Cantor-type construction used in defining the mapping. We start with an interval $I_{n_{0}}^{\prime}$ of length $2 r_{n_{0}}$. Divide $I_{n_{0}}^{\prime}$ into $2^{n}$ 
intervals of length $2 R_{n_{0}+1}$. We denote these intervals $I_{i, n_{0}+1}$, where $i=1,2, \ldots, 2^{n}$, and denote the corresponding midpoints of these intervals by $a_{i, n_{0}+1}$. Define also intervals $I_{i, n_{0}+1}^{\prime}$ with the same centers and lengths $2 r_{n_{0}+1}$. To ensure that $R_{j}>r_{j}$, we shall choose $n_{0}$ large enough. We use also the following notation $A_{i, n_{0}+1}:=I_{i, n_{0}+1} \backslash I_{i, n_{0}+1}^{\prime}$. We continue inductively. Assuming that we have defined the intervals $I_{i, j}^{\prime}$ with lengths $2 r_{j}, i=1,2, \ldots, 2^{n\left(j-n_{0}\right)}$, we proceed dividing each interval $I_{i, j}^{\prime}$ into $2^{n}$ intervals of length $2 R_{j+1}$. These intervals are denoted by $I_{i^{\prime}, j+1}$, and their midpoints are $a_{i^{\prime}, j+1}$, where $i^{\prime}=1,2, \ldots, 2^{n\left(j+1-n_{0}\right)}$. Again, we choose the intervals $I_{i^{\prime}, j+1}^{\prime}$ with midpoints $a_{i^{\prime}, j+1}$ and lengths $2 r_{j+1}$, and define $A_{i^{\prime}, j+1}:=I_{i^{\prime}, j+1} \backslash I_{i^{\prime}, j+1}^{\prime}$.

Let $d>0$ satisfy $2^{n} d^{v}=1$. For each $j \in \mathbb{N}$ we define $\varphi_{j}:\left[0, \infty\left[\rightarrow\left[0, d^{j}\right]\right.\right.$ by

$$
\varphi_{j}(r)= \begin{cases}d^{j}, & r \leq r_{j} \\ d^{j} \frac{\log \frac{R_{j}}{r}}{\log \frac{R_{j}}{r_{j}}}, & r_{j}<r<R_{j} \\ 0, & r \geq R_{j} .\end{cases}
$$

For the derivative of $\varphi_{j}, j \in \mathbb{N}$ and $j \geq n_{0}$, we have the estimate

$$
\left|\varphi_{j}^{\prime}(r)\right| \leq d^{j}\left(\log \frac{R_{j}}{r_{j}}\right)^{-1} \frac{1}{r} \leq C d^{j} j^{1-\beta} \frac{1}{r},
$$

for $r_{j}<r<R_{j}$, since for large $j$ we have

$$
\log \frac{R_{j}}{r_{j}}=(j+1)^{\beta}-j^{\beta}-n \log 2 \geq \beta j^{\beta-1}-n \log 2 \geq C j^{\beta-1} .
$$

Let $e_{1}, e_{2}, \ldots, e_{n}$ be the standard basis of $\mathbb{R}^{n}$. Let $v_{i}, i=1,2, \ldots, 2^{n}$, be the elements of the set $\left\{\sum_{k=1}^{n} a_{k} e_{k}: a_{k}= \pm 1\right\}$ in some fixed order. For all integers $k>2^{n}$ we define $v_{k}=v_{k-2^{n}}$.

For all indices $j=n_{0}+1, n_{0}+2, \ldots$ and $i=1,2, \ldots, 2^{n\left(j-n_{0}\right)}$ and all $x \in I_{n_{0}}^{\prime}$ we define $\tilde{\varphi}_{i j}(x)=(1-d) v_{i} \varphi_{j}\left(\left|x-a_{i, j}\right|\right)$. In addition, we set

$$
f_{j}=\sum_{i=1}^{2^{n\left(j-n_{0}\right)}} \tilde{\varphi}_{i j}
$$

Finally, we define

$$
f=\sum_{j=n_{0}+1}^{\infty} f_{j} .
$$

It is clear that the above sum converges at every point.

In the following subsections we prove (21) and establish the correct modulus of continuity, but first, we explain why $\mathcal{H}^{v}\left(f\left(I_{n_{0}}^{\prime}\right)\right)>0$. More precisely, we show that $f\left(I_{n_{0}}^{\prime}\right)$ contains a Cantor set with positive $v$-dimensional Hausdorff measure. Define $Q_{j}^{i} j=n_{0}+1, n_{0}+2, \ldots$ and $i=1, \ldots, 2^{n\left(j-n_{0}\right)}$, to be the closed cube with sidelength $d^{j}$, centre $f\left(a_{i, j}\right)$ and sides parallel to the coordinate axes. The set $C=\cap_{j=n_{0}+1}^{\infty} \cup_{i=1}^{2^{n\left(j-n_{0}\right)}} Q_{j}^{i}$ is a Cantor set in $\mathbb{R}^{n}$ with $\mathcal{H}^{v}(C)>0$, see $\left[2\right.$, Theorem 8.6] or $[13,4.13]$. Since $f\left(I_{n_{0}}^{\prime}\right)$ contains the centres of each $Q_{j}^{i}$, it must also contain the set $C$ by continuity. 
4.2. Finiteness of energy. Next, we show that for every $x \in A_{i, j}$ with fixed $j \geq n_{0}$ and $i \in\left\{1, \ldots, 2^{n\left(j-n_{0}\right)}\right\}$, the mapping $f$ satisfies the estimate

$$
\begin{aligned}
& \int_{I_{n_{0}}^{\prime}} \frac{|f(x)-f(y)|^{v}}{|x-y|^{2}} \log ^{\lambda}\left(e+\frac{|f(x)-f(y)|}{|x-y|}\right) d y \\
& \quad \leq C 2^{n\left(-j+n_{0}\right)}\left(\log \frac{R_{j}}{r_{j}}\right)^{-v} \frac{1}{\left|x-a_{i, j}\right|} \log ^{\lambda}\left(\frac{1}{\left|x-a_{i, j}\right|}\right)+C_{n_{0}}
\end{aligned}
$$

with some positive constants $C, C_{n_{0}}$, independent of $x, i$ and $j$. To prove this, we split the interval $I_{n_{0}}^{\prime}$ into a finite number of pieces and show the estimate for each of these pieces separately. This is done in the following subsections. The estimate (24) then follows by summing all the obtained estimates together. We first take care of the case $\lambda \geq 0$ and then indicate the required changes for $\lambda<0$.

Fix $x \in A_{i, j}$. We may assume that $a_{i, j}=0$ and that $x \geq 0$. We also ensure that $n_{0}$ is large enough to guarantee $r_{j}<R_{j} / 2$. Additionally, notice that

$$
\log \frac{1}{R_{j}} \leq \log \frac{1}{x} \leq \log \frac{1}{r_{j}}
$$

and

$$
C^{-1} j^{\beta} \leq(j-1)^{\beta} \leq(j+1)^{\beta} \leq C j^{\beta} .
$$

Both of these estimates are elementary and we will use them without an explicit mention.

4.3. Points close to $x$. Here, we integrate over the interval

$$
[a, b]:=\left[\max \left\{x / 2, \gamma r_{j}\right\}, \min \left\{2 x, 3 R_{j} / 2, \zeta\right\}\right],
$$

where $\gamma=\frac{2^{n+1}-1}{2^{n+1}}$ and $\zeta$ is the right endpoint of $I_{n_{0}}^{\prime}$. We assume that $n_{0}$ is so large that $\left|\varphi_{k+1}^{\prime}\left(R_{k+1}\right)\right| \geq\left|\varphi_{k}^{\prime}\left(r_{k}\right)\right|$ for all $k=n_{0}+1, \ldots$ If $x / 2<r_{j}$, we estimate, using (23),

$$
\frac{|f(x)-f(y)|}{|x-y|} \leq\left|\varphi_{j+1}^{\prime}\left(\frac{R_{j+1}}{2}\right)\right| \leq d^{j+1} \frac{2}{R_{j+1}} \log ^{-1} \frac{R_{j+1}}{r_{j+1}} \leq C d^{j} \frac{1}{x} \log ^{-1} \frac{R_{j}}{r_{j}} \leq \frac{1}{x}-e
$$

for each $y \in[a, b]$ and large enough $j$. Otherwise,

$$
\frac{|f(x)-f(y)|}{|x-y|} \leq\left|\varphi_{j}^{\prime}\left(\frac{x}{2}\right)\right| \leq d^{j} \frac{2}{x} \log ^{-1} \frac{R_{j}}{r_{j}} \leq \frac{1}{x}-e .
$$

Therefore, we have

$$
\begin{aligned}
\int_{[a, b]} \frac{|f(x)-f(y)|^{v}}{|x-y|^{2}} \log ^{\lambda}\left(e+\frac{|f(x)-f(y)|}{|x-y|}\right) d y & \leq C d^{j v}\left(\log \frac{R_{j}}{r_{j}}\right)^{-v} \frac{1}{x^{v}} \log ^{\lambda}\left(\frac{1}{x}\right) \int_{[0,2 x]}|x-y|^{v-2} d y \\
& \leq C 2^{-j n}\left(\log \frac{R_{j}}{r_{j}}\right)^{-v} \frac{1}{x} \log ^{\lambda}\left(\frac{1}{x}\right),
\end{aligned}
$$

as desired. For the remaining points $y \in I_{n_{0}}^{\prime} \backslash[a, b]$, we have $|x-y|>2^{-n-1} x$. 
4.4. Parent set and its descendants. In this subsection we integrate over the set $I_{i^{\prime}, j-1} \backslash[a, b]$, where $i^{\prime}$ is such that $A_{i, j} \subset I_{i^{\prime}, j-1}^{\prime}$. This set consists of $A_{i, j} \backslash[a, b]$, of its parent set $A_{i^{\prime}, j-1} \backslash[a, b]$, of its $2^{n}-1$ siblings $A_{i^{\prime \prime}, j} \backslash[a, b]$, contained in $I_{i^{\prime}, j-1}^{\prime}$, and of $2^{n}$ intervals $I_{i^{\prime \prime}, j}^{\prime}$ and $I_{i, j}^{\prime} \backslash[a, b]$ of length $2 r_{j}$, embraced by those $A_{i^{\prime \prime}, j}$ and $A_{i, j}$. In the case $j=n_{0}+1$ the only difference is the absence of the set $A_{i^{\prime}, j-1} \backslash[a, b]$. For a point $y$ in any of these sets, we have

$$
\log ^{\lambda}\left(e+\frac{|f(x)-f(y)|}{|x-y|}\right) \leq C \log ^{\lambda} \frac{1}{x}
$$

thus, it remains to estimate the integral of the quotient $|f(x)-f(y)|^{v} /|x-y|^{2}$. This is done differently for each of the mentioned sets.

\subsubsection{Interval containing $x$. For $y \in A_{i, j} \backslash[a, b]$ we have the estimate}

$$
|f(x)-f(y)| \leq \sqrt{n} d^{j}\left(\log \frac{R_{j}}{r_{j}}\right)^{-1}\left|\log \frac{R_{j}}{x}-\log \frac{R_{j}}{|y|}\right| \leq \sqrt{n} d^{j}\left(\log \frac{R_{j}}{r_{j}}\right)^{-1}\left|\log \frac{|y|}{x}\right|
$$

Furthermore,

$$
\int_{A_{i, j} \backslash[a, b]} \frac{\left|\log \frac{|y|}{x}\right|^{v}}{|x-y|^{2}} d y=\frac{1}{x} \int_{\left.A_{i, j} \backslash a, b\right]} \frac{\left|\log \frac{|y|}{x}\right|^{v}}{\left.|1-y| x\right|^{2}} \frac{d y}{x} \leq \frac{1}{x}\left(\int_{-\infty}^{\frac{1}{2}} \frac{|\log | t||^{v}}{(1-t)^{2}} d t+\int_{2}^{\infty} \frac{|\log t|^{v}}{(1-t)^{2}} d t\right) \leq \frac{C}{x} .
$$

Combining this estimate with (26) and (27), we obtain the desired estimate.

4.4.2. Siblings of $A_{i, j}$. Let us fix an index $i^{\prime \prime}$, so that both $A_{i, j}$ and $A_{i^{\prime \prime}, j}$ are contained in the same interval $I_{i^{\prime}, j-1}^{\prime}$ of generation $j-1$. Then

$$
\begin{aligned}
|f(x)-f(y)| \leq\left|f(x)-f\left(R_{j}\right)\right|+\left|f(y)-f\left(R_{j}\right)\right| & \leq \sqrt{n} d^{j}\left(\log \frac{R_{j}}{r_{j}}\right)^{-1}\left(\log \frac{R_{j}}{x}+\log \frac{R_{j}}{\left|y-a_{i^{\prime \prime}, j}\right|}\right) \\
& =\sqrt{n} d^{j}\left(\log \frac{R_{j}}{r_{j}}\right)^{-1} \log \frac{R_{j}^{2}}{x\left|y-a_{i^{\prime \prime}, j}\right|}
\end{aligned}
$$

for each $y \in A_{i^{\prime \prime}, j} \backslash[a, b]$. Note also that $|x-y| \geq R_{j} / 4$ for each of these $y$. Hence, substituting $t=\frac{x\left|y-a_{i^{\prime \prime}, j}\right|}{R_{j}^{2}}$ in each of the two intervals, we obtain

$$
\int_{A_{i^{\prime \prime}, j} \backslash[a, b]} \frac{\left(\log \frac{R_{j}^{2}}{x\left|y-a_{i^{\prime \prime}, j}\right|}\right)^{v}}{|x-y|^{2}} d y \leq \frac{16}{R_{j}^{2}} \int_{A_{i^{\prime \prime}, j}}\left(\log \frac{R_{j}^{2}}{x\left|y-a_{i^{\prime \prime}, j}\right|}\right)^{v} d y \leq \frac{C}{x} \int_{0}^{1}|\log t|^{v} d t \leq \frac{C}{x} .
$$

The desired estimate follows from the last inequality, (26) and (28). Note that there are $2^{n}-1$ suitable $i^{\prime \prime}$. 
4.4.3. Children of $A_{i, j}$. We consider one of $2^{n}$ sets $A_{i_{0}, j+1}$, such that $A_{i_{0}, j+1} \subset I_{i, j}^{\prime}$. If $y \in A_{i_{0}, j+1} \backslash$ $[a, b]$, we have

$$
\begin{aligned}
|f(x)-f(y)| & \leq\left|f(x)-f\left(r_{j}\right)\right|+\left|f(y)-f\left(r_{j}\right)\right| \leq \sqrt{n} d^{j}\left(1-\frac{\log \frac{R_{j}}{x}}{\log \frac{R_{j}}{r_{j}}}\right)+\sqrt{n} d^{j+1} \frac{\log \frac{R_{j+1}}{\left|y-a_{i_{0}, j+1}\right|}}{\log \frac{R_{j+1}}{r_{j+1}}} \\
& \leq \sqrt{n} d^{j}\left(\log \frac{R_{j}}{r_{j}}\right)^{-1}\left[\log \frac{R_{j}}{r_{j}}-\log \frac{R_{j}}{x}+\log \frac{R_{j+1}}{\left|y-a_{i_{0}, j+1}\right|}\right] \\
& \leq \sqrt{n} d^{j}\left(\log \frac{R_{j}}{r_{j}}\right)^{-1} \log \frac{x}{2^{n}\left|y-a_{i_{0}, j+1}\right|}
\end{aligned}
$$

Using the inequality $|x-y| \geq 2^{-n-1} x$ for $y \notin[a, b]$, we further estimate with the substitute $t=\frac{2^{n}\left|y-a_{i 0, j+1}\right|}{x}$ :

$$
\int_{A_{i_{0}, j+1} \backslash[a, b]} \frac{\left(\log \frac{x}{2^{n}\left|y-a_{i_{0}, j+1}\right|}\right)^{v}}{|x-y|^{2}} d y \leq \frac{2^{2 n+2}}{x} \int_{A_{i_{0}, j+1}}\left(\log \frac{x}{2^{n}\left|y-a_{i_{0}, j+1}\right|}\right)^{v} \frac{d y}{x} \leq \frac{C}{x} \int_{0}^{1}|\log t|^{v} d t \leq \frac{C}{x} .
$$

Together with (26) and (29), this gives us (24).

4.4.4. Parent of $A_{i, j}$. We fix the unique $i^{\prime}$ with $A_{i, j} \subset I_{i^{\prime}, j-1}^{\prime}$. Similarly to (29), interchanging the roles of $y$ and $x$ and shifting the index, we obtain

$$
\begin{aligned}
|f(x)-f(y)| & \leq\left|f(x)-f\left(R_{j}\right)\right|+\left|f(y)-f\left(R_{j}\right)\right| \leq \sqrt{n} d^{j-1}\left(\log \frac{R_{j-1}}{r_{j-1}}\right)^{-1} \log \frac{\left|y-a_{i^{\prime}, j-1}\right|}{2^{n} x} \\
& \leq C d^{j}\left(\log \frac{R_{j}}{r_{j}}\right)^{-1} \log \frac{\left|y-a_{i^{\prime}, j-1}\right|}{2^{n} x} \leq C d^{j}\left(\log \frac{R_{j}}{r_{j}}\right)^{-1} \log \frac{2|y|}{x}
\end{aligned}
$$

for $y \in A_{i^{\prime}, j-1} \backslash[a, b]$, since $\left|a_{i^{\prime}, j-1}\right| \leq r_{j-1}$ and $|y| \geq R_{j}$. We also have $y \geq 3 x / 2$, when $y>0$. Therefore,

$$
\int_{A_{i^{\prime}, j-1} \backslash[a, b]} \frac{\left(\log \frac{2|y|}{x}\right)^{v}}{|x-y|^{2}} d y=\frac{1}{x} \int_{A_{i^{\prime}, j-1} \backslash[a, b]} \frac{\left(\log \frac{2|y|}{x}\right)^{v}}{\left.|1-y| x\right|^{2}} \frac{d y}{x} \leq \frac{1}{x}\left(\int_{-\infty}^{-1} \frac{\log ^{v}|2 t|}{(1-t)^{2}} d t+\int_{\frac{3}{2}}^{\infty} \frac{\log ^{v}(2 t)}{(1-t)^{2}} d t\right) \leq \frac{C}{x} .
$$

The conclusion is as in the previous subsections.

4.4.5. Grandchildren of $A_{i, j}$, nephews of $A_{i, j}$ and their descendants. As before, we fix $i_{0}$ with $A_{i_{0}, j+1} \subset I_{i, j}^{\prime}$. Let us integrate over the set $I_{i_{0}, j+1}^{\prime}$. Here we use the fast decay of the numbers $r_{j}$. The estimate is the following:

$$
\int_{I_{i_{0}, j+1}^{\prime}} \frac{|f(x)-f(y)|^{v}}{|x-y|^{2}} d y \leq C d^{j v}\left(\log \frac{R_{j}}{r_{j}}\right)^{-v} \frac{1}{x} \frac{r_{j+1}}{x}\left(\log \frac{R_{j}}{r_{j}}\right)^{v} \leq C 2^{-j n}\left(\log \frac{R_{j}}{r_{j}}\right)^{-v} \frac{1}{x^{\prime}}
$$

since

$$
\begin{aligned}
\frac{r_{j+1}}{x}\left(\log \frac{R_{j}}{r_{j}}\right)^{v} \leq \frac{r_{j+1}}{r_{j}}\left(\log \frac{R_{j}}{r_{j}}\right)^{v} & \leq C j^{v(\beta-1)} \exp \left(-(j+2)^{\beta}+(j+1)^{\beta}\right) \\
& \leq C j^{v(\beta-1)} \exp \left(-\beta(j+1)^{\beta-1}\right) \leq C .
\end{aligned}
$$


The estimate (24) follows from (26).

Similar computations imply the conclusion for the intervals $I_{i^{\prime \prime}, j}^{\prime}$ such that $A_{i, j} \cup A_{i^{\prime \prime}, j} \subset I_{i^{\prime}, j-1}^{\prime}$ for $i^{\prime}$ as above; because $|x-y| \geq R_{j}$ for $y \in I_{i^{\prime \prime}, j}^{\prime}$ and $\left|I_{i^{\prime \prime}, j}^{\prime}\right|=2 r_{j}$.

4.5. Earlier generations. Now we integrate over the set $I_{n_{0}}^{\prime} \backslash I_{i^{\prime}, j-1}$ where $I_{i^{\prime}, j-1} \supset I_{i, j}$. Note that if $y \in\left[R_{j}+\sum_{i=k+1}^{j-1}\left(R_{i}-r_{i}\right), R_{j}+\sum_{i=k}^{j-1}\left(R_{i}-r_{i}\right)\right]$ for some $k \leq j-2$, then there is an interval $I_{l, k}$ of generation $k$, which contains both $x$ and $y$. This implies $|f(x)-f(y)| \leq 2 \sqrt{n} d^{k}$.

Define numbers $A_{k}=\sum_{i=k+1}^{j-1}\left(R_{i}-r_{i}\right)$ and $B_{k}=\sum_{i=k}^{j-1}\left(R_{i}-r_{i}\right)$. We choose $n_{0} \in \mathbb{N}$ so large that the inequality

$$
e+\sqrt{n} \frac{2^{-l+1}}{A_{l}} \leq A_{l}^{-1}
$$

holds for each $l \geq n_{0}$.

If $j=n_{0}+1$, there are no intervals of earlier generations; and if $j=n_{0}+2$, then the only intervals of earlier generations are the intervals of the generation $n_{0}+1$, which are treated in (34) and in Section 4.4.4. Using (31), we get the following estimate for all $j \geq n_{0}+3$ :

$$
\begin{aligned}
& \int_{\left[R_{j}+\left(R_{j-1}-r_{j-1}\right), R_{j}+\sum_{i=n_{0}+1}^{j-1}\left(R_{i}-r_{i}\right)\right]} \frac{|f(x)-f(y)|^{v}}{|x-y|^{2}} \log ^{\lambda}\left(e+\frac{|f(x)-f(y)|}{|x-y|}\right) d y \\
& \leq C \sum_{k=n_{0}+1}^{j-2} \int_{\left[R_{j}+A_{k}, R_{j}+B_{k}\right]} \frac{d^{k v}}{|x-y|^{2}} \log ^{\lambda}\left(A_{k}^{-1}\right) d y \leq C \sum_{k=n_{0}+1}^{j-2} 2^{-k n} \log ^{\lambda}\left(A_{k}^{-1}\right) \int_{A_{k}}^{\infty} \frac{d t}{t^{2}} \\
& \leq C \sum_{k=n_{0}+1}^{j-2} 2^{-k n} \log ^{\lambda}\left(A_{k}^{-1}\right) \frac{1}{A_{k}} \leq C \sum_{k=n_{0}}^{j-2} 2^{-k n} \log ^{\lambda}\left(R_{k+1}^{-1}\right) \frac{1}{R_{k+1}} .
\end{aligned}
$$

Next, we show that for all large $k$ we have

$$
2^{-k n} \log ^{\lambda}\left(R_{k}^{-1}\right) \frac{1}{R_{k}} \geq 2 \cdot 2^{(-k+1) n} \log ^{\lambda}\left(R_{k-1}^{-1}\right) \frac{1}{R_{k-1}}
$$

This is rather easy:

$$
\frac{2^{-k n} \log ^{\lambda}\left(R_{k}^{-1}\right) \frac{1}{R_{k}}}{2^{(-k+1) n} \log ^{\lambda}\left(R_{k-1}^{-1}\right) \frac{1}{R_{k-1}}} \geq \frac{1}{2^{n}} \exp \left(k^{\beta}-(k-1)^{\beta}\right)\left(\frac{k}{k-1}\right)^{\beta \lambda} \geq \frac{1}{2^{n}} \exp \left(\beta(k-1)^{\beta-1}\right) \geq 2,
$$

as soon as $k$ is large enough. Now the sum on the right hand side of (32) is seen to be smaller than

$$
C \sum_{k=n_{0}}^{j-2} 2^{k-j+2} 2^{-j n} \log ^{\lambda}\left(R_{j-1}^{-1}\right) \frac{1}{R_{j-1}} \leq C 2^{-j n} \log ^{\lambda}\left(R_{j-1}^{-1}\right) \frac{1}{R_{j-1}}
$$


Besides, similarly to (30), we observe that $\frac{R_{j}}{R_{j-1}} \log ^{v} \frac{R_{j}}{r_{j}} \leq C$, which implies the desired estimate

$$
\begin{gathered}
\int_{\left[R_{j}+\left(R_{j-1}-r_{j-1}\right), R_{j}+\sum_{i=n_{0}+1}^{j-1}\left(R_{i}-r_{i}\right)\right]} \frac{|f(x)-f(y)|^{v}}{|x-y|^{2}} \log ^{\lambda}\left(e+\frac{|f(x)-f(y)|}{|x-y|}\right) d y \\
\leq C 2^{-j n} \log ^{\lambda}\left(\frac{1}{x}\right) \log ^{-v} \frac{R_{j}}{r_{j}} \frac{1}{x} .
\end{gathered}
$$

The integral over the set $\left[-R_{j}-B_{n_{0+1}},-R_{j}-\left(R_{j-1}-r_{j-1}\right)\right]$ is estimated in the same way as the integral over the set $\left[R_{j}+\left(R_{j-1}-r_{j-1}\right), R_{j}+B_{n_{0+1}}\right]$, since we have the same estimates for $|f(x)-f(y)|$ and $|x-y|$.

The interval $\left[-R_{j}-B_{n_{0}+1}, R_{j}+B_{n_{0}+1}\right]$ does not cover $I_{n_{0}}^{\prime}$. It remains to estimate the integral over the set $I_{n_{0}}^{\prime} \backslash\left[-R_{j}-B_{n_{0}+1}, R_{j}+B_{n_{0}+1}\right]$ :

$$
\begin{aligned}
& \int_{I_{n_{0}}^{\prime} \backslash\left[-R_{j}-B_{n_{0}+1}, R_{j}+B_{n_{0}+1}\right]} \frac{|f(x)-f(y)|^{v}}{|x-y|^{2}} \log ^{\lambda}\left(e+\frac{|f(x)-f(y)|}{|x-y|}\right) d y \\
& \leq C\left|I_{n_{0}}^{\prime}\right| B_{n_{0}+1}^{-2} \log ^{\lambda}\left(e+B_{n_{0}+1}^{-1}\right) \leq C_{n_{0}} .
\end{aligned}
$$

4.6. Case $\lambda<0$. We have to make several changes to above arguments. Our aim is to divide the points in $I_{n_{0}}^{\prime}$ into two sets so that we can estimate the logarithmic term on the left hand side of (24).

Let $I_{i^{\prime}, j-1}$ be the interval of generation $j-1$ which contains $A_{i, j}$. The length of this interval is at most $2 R_{j-1}$. First, we consider all the points $y \in I_{i^{\prime}, j-1}$ such that

$$
\frac{|f(x)-f(y)|}{|x-y|} \geq R_{j-1}^{-\frac{v-1}{v}}
$$

Notice that the logarithms of $r_{j}$ and $R_{j-1}$ are comparable, then by (25) and (35) we have

$$
\log \frac{|f(x)-f(y)|}{|x-y|} \geq C \log \frac{1}{R_{j-1}} \geq C \log \frac{1}{r_{j}} \geq C \log \frac{1}{x} .
$$

Using this to estimate the logarithm on the set of points satisfying (35) we obtain the analogues of estimates in Sections 4.3 and 4.4.

For the points $y \in I_{i^{\prime}, j-1}$, for which (35) is not satisfied, we have the estimate

$$
\int_{\left\{y \in I_{i^{\prime}, j-1}:(35) \text { is not true }\right\}} \frac{|f(x)-f(y)|^{v}}{|x-y|^{2}} \log ^{\lambda}\left(e+\frac{|f(x)-f(y)|}{|x-y|}\right) d y \leq C .
$$

When considering the earlier generations in Section 4.5, we had to estimate the sum

$$
\sum_{k=n_{0}+1}^{j-2} \int_{\left[R_{j}+\sum_{i=k+1}^{j-1}\left(R_{i}-r_{i}\right), R_{j}+\sum_{i=k}^{j-1}\left(R_{i}-r_{i}\right)\right]} \frac{|f(x)-f(y)|^{v}}{|x-y|^{2}} \log ^{\lambda}\left(e+\frac{|f(x)-f(y)|}{|x-y|}\right) d y .
$$


Again, for $\lambda<0$ we split the integration intervals into two sets. We use the notation introduced in Subsection 4.5. In the set of points $y \in\left[R_{j}+A_{k}, R_{j}+B_{k}\right]$, where

$$
\frac{|f(x)-f(y)|}{|x-y|} \leq R_{k}^{-\frac{v-1}{2 v}}
$$

the sum (36) reduces to

$$
C \sum_{k=n_{0}+1}^{j-2} R_{k}^{\frac{v-1}{2}} \leq C \sum_{k=1}^{j-2} \exp \left(-\frac{v-1}{2} k^{\beta}\right) \leq C .
$$

On the other hand, on the set of points $y$, such that

$$
\frac{|f(x)-f(y)|}{|x-y|}>R_{k}^{-\frac{v-1}{2 v}},
$$

we may estimate the logarithm term in (36) from above and then use the same arguments as in Section 4.5 to obtain the desired estimate.

4.7. $f$ has finite energy. Now we show that the required energy is finite. If $-1<\lambda<v-1$, then using (24) we obtain the estimate

$$
\begin{aligned}
\int_{I_{n_{0}}} & \int_{I_{n_{0}}} \frac{|f(x)-f(y)|^{v}}{|x-y|^{2}} \log ^{\lambda}\left(e+\frac{|f(x)-f(y)|}{|x-y|}\right) d y d x \\
& \leq \sum_{j=n_{0}+1}^{\infty} \sum_{i=1}^{2^{\left(j-n_{0}\right) n}} \int_{A_{i, j}} \int_{I_{n_{0}}^{\prime}} \frac{|f(x)-f(y)|^{v}}{|x-y|^{2}} \log ^{\lambda}\left(e+\frac{|f(x)-f(y)|}{|x-y|}\right) d y d x \\
& \leq C \sum_{j=n_{0}+1}^{\infty} \sum_{i=1}^{2^{\left(j-n_{0}\right) n}} \int_{A_{i, j}} 2^{-j n}\left(\log \frac{R_{j}}{r_{j}}\right)^{-v} \frac{1}{\left|x-a_{i, j}\right|} \log ^{\lambda} \frac{1}{\left|x-a_{i, j}\right|}+C_{n_{0}} d x \\
& \leq C \sum_{j=n_{0}+1}^{\infty} \sum_{i=1}^{2^{\left(j-n_{0}\right) n}} 2^{-j n}\left(\log \frac{R_{j}}{r_{j}}\right)^{-v}\left(\log ^{\lambda+1} \frac{1}{\left|r_{j}\right|}-\log ^{\lambda+1} \frac{1}{\left|R_{j}\right|}\right)+C_{n_{0}}\left|I_{n_{0}}^{\prime}\right| \\
& \leq C \sum_{j=n_{0}+1}^{\infty}\left(\log \frac{R_{j}}{r_{j}}\right)^{-v}\left(\log { }^{\lambda+1} \frac{1}{\left|r_{j}\right|}-\log ^{\lambda+1} \frac{1}{\left|R_{j}\right|}\right)+C_{n_{0}} \\
& \leq C \sum_{j=n_{0}+1}^{\infty} j^{-v(\beta-1)}\left(\log ^{\lambda+1}\left(\exp \left((j+1)^{\beta}\right)\right)-\log ^{\lambda+1}\left(\exp \left(j^{\beta}\right)\right)\right)+C_{n_{0}} \\
& \leq C \sum_{j=n_{0}+1}^{\infty} j^{\nu-v \beta}\left(\left((j+1)^{\beta(\lambda+1)}\right)-\left(j^{\beta(\lambda+1)}\right)\right)+C_{n_{0}} \leq C \sum_{j=n_{0}+1}^{\infty} j^{\nu-1-v \beta+\beta(\lambda+1)}+C_{n_{0}} \\
& <\infty,
\end{aligned}
$$

if $v-1+\beta(\lambda-v+1)<-1$, that is $1 / \beta<\frac{v-1-\lambda}{v}$. Using a linear approximation for the logarithm in a calculation similar to the one above, we obtain the same result also for $\lambda=-1$. 
4.8. Modulus of continuity. We will show that the mapping $f$ has a modulus of continuity $\varphi(t)=C^{\prime} \exp \left(-C \log ^{1 / \beta} \frac{1}{t}\right)$, where $C$ and $C^{\prime}$ are some positive constants. First, let $x, y \in$ $\left[a_{i, j}+r_{j}, a_{i, j}+R_{j}\right]$ for some $j \in\left\{n_{0}+1, \ldots\right\}$ and $i \in\left\{1, \ldots, 2^{n\left(j-n_{0}\right)}\right\}$. We may assume again that $a_{i, j}=0$. Then

$$
|f(x)-f(y)|=(1-d) \sqrt{n} \frac{d^{j}}{\log \frac{R_{j}}{r_{j}}}\left|\log \frac{x}{y}\right| .
$$

Assume that $x>y$ and $d^{m-1} r_{j} \leq x-y<d^{m} r_{j}$ for some $m \in \mathbb{Z}$. If $m \geq 0$, we get

$$
\begin{aligned}
|f(x)-f(y)| & =(1-d) \sqrt{n} \frac{d^{j}}{\log \frac{R_{j}}{r_{j}}}\left|\log \left(1+\frac{x-y}{y}\right)\right| \leq C \frac{d^{j}}{\log \frac{R_{j}}{r_{j}}} \frac{x-y}{r_{j}} \leq C \frac{d^{j}}{\log \frac{R_{j}}{r_{j}}} d^{m} \\
& \leq C \frac{d^{j+m}}{\log \frac{R_{j}}{r_{j}}} \exp \left(C_{0}\left(\log \frac{1}{|x-y|}\right)^{1 / \beta}\right) \exp \left(-C_{0}\left(\log \frac{1}{|x-y|}\right)^{1 / \beta}\right),
\end{aligned}
$$

where we fix $\left.C_{0} \in\right] 0, \min \left\{\log d^{-1},\left(\log d^{-1}\right)^{1-1 / \beta}\right\}[$. In addition, when $j$ is large enough, we have

$$
\begin{aligned}
\frac{d^{j+m}}{\log \frac{R_{j}}{r_{j}}} & \exp \left(C_{0}\left(\log \frac{1}{|x-y|}\right)^{1 / \beta}\right) \leq \frac{d^{j+m}}{C j^{\beta-1}} \exp \left(C_{0}\left(\log \frac{d^{-m+1}}{r_{j}}\right)^{1 / \beta}\right) \\
& \leq C d^{j+m} \exp \left(C_{0}\left((-m+1) \log d+(j+1)^{\beta}\right)^{1 / \beta}\right) \\
& \leq C d^{j+m} \exp \left(C_{0} m^{1 / \beta} \log ^{1 / \beta} d^{-1}+C_{0} j+C_{0}\right) \\
& \leq C \exp \left(m \log d+C_{0} m^{1 / \beta} \log ^{1 / \beta} d^{-1}+\left(C_{0}+\log d\right) j\right) \\
& \leq C \exp \left(m \log d+C_{0} m^{1 / \beta} \log ^{1 / \beta} d^{-1}\right) \exp \left(\left(C_{0}+\log d\right) j\right) \leq 1,
\end{aligned}
$$

by the choice of $C_{0}$ and the fact that $\beta>1$.

If $m<0$, then (37) implies

$$
\begin{aligned}
|f(x)-f(y)| & =C \frac{d^{j}}{\log \frac{R_{j}}{r_{j}}} \log \left(1+\frac{x-y}{y}\right) \leq C d^{j} j^{1-\beta} \log \left(1+\frac{x-y}{r_{j}}\right) \leq C d^{j} j^{1-\beta} \log \left(1+d^{m}\right) \\
& \leq C 2^{-j} j^{1-\beta}|m| .
\end{aligned}
$$

By the choice of $m$, we have

$$
\frac{R_{j}}{r_{j}} \geq d^{m-1}
$$

which yields

$$
|m| \leq C j^{\beta-1},
$$

and

$$
\log \frac{1}{|x-y|} \leq 2^{\beta} j^{\beta}
$$


These estimates with (38) give

$$
|f(x)-f(y)| \leq C^{\prime} \exp \left(-\frac{\log 2}{2}\left(\log \frac{1}{|x-y|}\right)^{1 / \beta}\right) .
$$

If $x$ and $y$ are in neighbouring intervals, then we have

$$
|f(x)-f(y)| \leq|f(x)-f(c)|+|f(c)-f(y)| \leq 2 C \exp \left(-C\left(\log \frac{1}{|x-y|}\right)^{1 / \beta}\right) .
$$

Here $c$ is the point where the two intervals meet.

If $x$ and $y$ are points which are not in same or neighbouring intervals, then there is the smallest $j$ such that a component interval of $A_{i, j}$ lies between $x$ and $y$. Thus $R_{j} / 2 \leq|x-y|$. That is, $-j \leq-C\left(\log \frac{1}{|x-y|}\right)^{1 / \beta}$. In this case, there is an interval $I_{i^{\prime}, j-1}$ (or $I_{n_{0}}^{\prime}$ in the case $j-1=n_{0}$ ) containing both $x$ and $y$. This implies $|f(x)-f(y)| \leq \sqrt{n} d^{j-1}$. Therefore, we get again the continuity estimate $|f(x)-f(y)| \leq d^{-1} \sqrt{n} \exp \left(-C\left(\log \frac{1}{|x-y|}\right)^{1 / \beta}\right)$.

4.9. Case $\lambda=v-1$. As before, this example is from [8], where it was presented in a slightly different context. We make the following modifications to the construction from the previous subsections. We define $R_{j}=\exp \left(-\exp \left(j^{\beta}\right)\right), r_{j}=2^{n} \exp \left(-\exp \left((j+1)^{\beta}\right)\right)$ and

$$
\varphi_{j}(r)= \begin{cases}d^{j}, & r \leq r_{j} \\ d^{j} \frac{\log \frac{\log \frac{1}{\log \frac{1}{R_{j}}}}{\log \frac{1}{r_{j}}},}{\log \frac{1}{\log \frac{1}{R_{j}}}} & r_{j}<r<R_{j} \\ 0, & r \geq R_{j},\end{cases}
$$

in place of (22).

Verification of the properties is similar to what is done in the previous subsections, but is more tedious. The details are left to the reader. When proving (21), instead of (24), one should establish

$$
\begin{aligned}
& \int_{I_{n_{0}}^{\prime}} \frac{|f(x)-f(y)|^{v}}{|x-y|^{2}} \log ^{\nu-1}\left(e+\frac{|f(x)-f(y)|}{|x-y|}\right) d y \\
& \leq C 2^{n\left(-j+n_{0}\right)}\left(\log \frac{\log \frac{1}{r_{j}}}{\log \frac{1}{R_{j}}}\right)^{-v} \frac{1}{\left|x-a_{i, j}\right|} \log ^{-1}\left(\frac{1}{\left|x-a_{i, j}\right|}\right)+C\left(n_{0}\right)
\end{aligned}
$$

for each $j=n_{0}+1, \ldots, i=1, \ldots, 2^{n\left(j-n_{0}\right)}$ and $x \in A_{i, j}$.

Acknowledgements. The authors thank the referee for the positive report.

\section{REFERENCES}

[1] Buckley, S. M. Space-filling curves and related functions. Irish Math. Soc. Bull., 36 (1996), 9-18.

[2] Falconer, K. J. The geometry of fractal sets, vol. 85 of Cambridge Tracts in Mathematics. Cambridge University Press, Cambridge, 1986.

[3] HajŁasz, P., And Tyson, J. T. Sobolev Peano cubes. Michigan Math. J. 56, 3 (2008), 687-702. 
[4] Hencl, S., Koskela, P., And Nieminen, T. Dimension gap under conformal mappings. Adv. Math. 230,3 (2012), 1423-1441.

[5] Howroyd, J. D. On the theory of Hausdorff measures in metric spaces. PhD thesis, University College London, 1994.

[6] Jaffard, S., And Nicolay, S. Pointwise smoothness of space-filling functions. Appl. Comput. Harmon. Anal. 26, 2 (2009), 181-199.

[7] Kauranen, A., And Koskela, P. Boundary blow-up under Sobolev mappings. Anal. PDE 7, 8 (2014), 1839-1850.

[8] Koskela, P., Malý, J., And Zürcher, T. Luzin's condition (N) and Sobolev mappings. Atti Accad. Naz. Lincei Cl. Sci. Fis. Mat. Natur. Rend. Lincei (9) Mat. Appl. 23, 4 (2012), 455-465.

[9] Koskela, P., and Nieminen, T. Quasiconformal removability and the quasihyperbolic metric. Indiana Univ. Math. J. 54, 1 (2005), 143-151.

[10] Koskela, P., and Rohde, S. Hausdorff dimension and mean porosity. Math. Ann. 309, 4 (1997), 593-609.

[11] Koskela, P., and Zapadinskaya, A. Dimension gap under Sobolev mappings. Adv. Math. 274 (2015), 385403.

[12] Malý, J., and Martio, O. Lusin's condition (N) and mappings of the class $W^{1, n}$. J. Reine Angew. Math. 458 (1995), 19-36.

[13] Mattila, P. Geometry of sets and measures in Euclidean spaces, vol. 44 of Cambridge Studies in Advanced Mathematics. Cambridge University Press, Cambridge, 1995. Fractals and rectifiability.

[14] Nieminen, T. Generalized mean porosity and dimension. Ann. Acad. Sci. Fenn. Math. 31, 1 (2006), $143-172$.

[15] Peano, G. Sur une courbe, qui remplit toute une aire plane. Math. Ann. 36, 1 (1890), 157-160.

[16] Sagan, H. Space-filling curves. Universitext. Springer-Verlag, New York, 1994.

[17] Shchepin, E. V. On fractal Peano curves. Tr. Mat. Inst. Steklova 247, Geom. Topol. i Teor. Mnozh. (2004), 294-303.

[18] Wildrick, K., AND Zürcher, T. Peano cubes with derivatives in a Lorentz space. Illinois J. Math. 53, 2 (2009), 365-378.

[19] Wildrick, K., AND Zürcher, T. Space filling with metric measure spaces. Math. Z. 270, 1-2 (2012), $103-131$.

\section{Aapo Kauranen}

Department of Mathematics and Statistics

University of Jyväskylä

P.O. Box 35 (MaD)

FI-40014

Finland

Pekka Koskela

Department of Mathematics and Statistics

University of Jyväskylä

P.O. Box 35 (MaD)

FI-40014

Finland

Aleksandra Zapadinskaya

Department of Mathematics

University of Pisa

Largo Bruno Pontecorvo 5

56127 Pisa

Italy

E-mail addresses: aapo.p.kauranen@jyu.fi, pkoskela@maths.jyu.fi, azapadinskaya@mail.dm.unipi.it 